\title{
Astrocytic Microdomains Confine A "molecular Memory" Enabling Long-Term Information Storage for Memory Consolidation
}

\section{Beatrice Vignoli}

University of Trento

\section{Gabriele Sansevero}

University of Florence

Manju Sasi

University Hospital Würzburg

Roberto Rimondini-Giorgini

University of Bologna

\section{Robert Blum}

University Hospital Würzburg

\section{Spartaco Santi}

National Research Council of Italy/IRCCS https://orcid.org/0000-0001-9856-7053

Nicoletta Berardi

University of Florence

Bai Lu

Tsinghua University

Marco Canossa ( $\square$ marco.canossa@unitn.it)

University of Trento

\section{Article}

Keywords: astrocytic, microdomains, molecular

Posted Date: January 28th, 2021

DOI: https://doi.org/10.21203/rs.3.rs-139639/v1

License: (c) (1) This work is licensed under a Creative Commons Attribution 4.0 International License. Read Full License

Version of Record: A version of this preprint was published at Communications Biology on October 5th, 2021. See the published version at https://doi.org/10.1038/s42003-021-02678-x. 



\section{Astrocytic microdomains confine a "molecular memory" enabling long- 2 term information storage for memory consolidation}

4 Beatrice Vignoli, ${ }^{1 *}$ Gabriele Sansevero, ${ }^{2}$ Manju Sasi, ${ }^{3}$ Roberto Rimondini-Giorgini, ${ }^{4}$

5 Robert Blum, ${ }^{3}$ Spartaco Santi, ${ }^{5}$ Nicoletta Berardi, ${ }^{6}$ Bai Lu, ${ }^{7}$ Marco Canossa $8, *$

$6{ }^{1}$ Department of Physics/Nanoscience Laboratory/Department of Cellular, Computational 7 and Integrative Biology (CIBIO) University of Trento, Povo (TN), 38123, Italy.

82 Department of Neuroscience, Psychology, Drug Research, Child Health 9 (NEUROFARBA), University of Florence, Florence, 50100, Italy/IRCCS Stella Maris 10 Foundation, 56128 Pisa, Italy.

113 Institute of Clinical Neurobiology, University Hospital Würzburg, Würzburg, 97078, 12 Germany.

134 Department of Medical and Surgical Sciences (DIMEC), University of Bologna, 14 Bologna, 40126, Italy.

$15{ }^{5}$ Institute of Molecular Genetics "Luigi Luca Cavalli-Sforza”, National Research Council 16 of Italy/IRCCS, Istituto Ortopedico Rizzoli, Bologna, 40136, Italy.

176 Department of Neuroscience, Psychology, Drug Research, Child Health 18 (NEUROFARBA), University of Florence, Florence, 50100, Italy.

$19{ }^{7}$ School of Pharmaceutical Sciences, Tsinghua University, Beijing, 100084, China.

$20{ }^{8}$ Department of Cellular, Computational and Integrative Biology (CIBIO) University of 21 Trento, Povo (TN), 38123, Italy.

$22 *$ Correspondence: marco.canossa@unitn.it; beatrice.vignoli@unitn.it 


\section{Abstract}

24 Memory consolidation requires astrocytic microdomains for protein recycling; but 25 whether this lays a mechanistic foundation for long-term information storage remains 26 enigmatic. Here we demonstrate that persistent synaptic strengthening invited astrocytic 27 microdomains to convert initially internalized (pro)-brain-derived neurotrophic factor 28 (proBDNF) into active prodomain (BDNFpro) and mature BDNF (mBDNF) for synaptic 29 re-use. While mBDNF activates TrkB, we uncovered a previously unsuspected function 30 for the cleaved BDNFpro, which increases TrkB/SorCS2 receptor complex at post31 synaptic sites. Astrocytic BDNFpro release reinforced TrkB phosphorylation to sustain 32 long-term synaptic potentiation and to retain memory in the novel object recognition 33 behavioral test. Thus, the switch from one inactive state to a multi-functional one of the 34 proBDNF provides post-synaptic changes that survive the initial activation (molecular 35 memory). This molecular asset confines local information storage in astrocytic 36 microdomains to selectively support memory circuits. 


\section{Introduction}

38 The most intriguing aspect of long-term potentiation (LTP) is the molecular basis 39 essential for its persistence, a suggestive hallmark for memory consolidation. Crick first 40 tackled the hypothesis that long-term memory storage is centered on a self-sustained 41 molecular adaptation, suggesting protein phosphorylation as a paradigm (1). Later on, the

42 discovery of in-side activation of CaMKII (2), confirmed the existence of "memory 43 molecules". CaMKII is initially activated by $\mathrm{Ca}^{2+}-$ Calmodulin. Due to a mechanism of 44 self-phosphorylation that makes the enzyme constitutively active, CaMKII no longer 45 requires the presence of $\mathrm{Ca}^{2+}-$ Calmodulin for its functionality $(3,4)$. More recently, 46 Kandel and co-workers reported on a different process by showing that prion-like 47 proteins (CPEB), aggregated in a self-perpetrating state are involved in the stabilization 48 of long-lasting synaptic changes through the control of local protein synthesis (5). Thus, a variety of proteins that no longer experiences the initial activation can maintain memory. In line with this rather simple but attractive model, the mechanism that converts a 51 transient neuronal stimulation into a persistent synaptic signaling during LTP has been a 52 long-standing question (6).

53 Neurons are exquisitely specialized for rapid electrical transmission of signals, but glial 54 cells, which do not communicate with electrical impulses are ideal for participating in 55 cognitive functions requiring long-term temporal regulation and broad spatial integration 56 (7-9). In the present study, we report that astrocytic membrane protrusions enwrapping 57 synaptic terminals (microdomains) are a previously unsuspected synaptic compartment 58 for the confinement of a "molecular memory". Once stimulated, neurons serve synthesis and release of brain-derived neurotrophic factor (BDNF) into the extracellular space (10), a key step in the induction of long-term synaptic modification (11). In addition, neurons

61 provide secretion of the precursor protein ( $32 \mathrm{kDa}$ proBDNF), consisting of prodomain

62 ( 12 kDa BDNFpro) and mature protein ( $\sim 14 \mathrm{kDa}$ mBDNF) (12). We have previously 63 reported that proBDNF is cleared by astrocytic microdomains via $\mathrm{p} 75^{\mathrm{NTR}}$-mediated 64 endocytosis (13-15) and recycled back to neurons for LTP stabilization and memory 65 retention (15). Here we demonstrated that astrocytic microdomains coordinate in 66 subsequent steps, (i) the processing of previously internalized proBDNF to generate 
67 BDNFpro and mBDNF products; (ii) the vesicular storage and (iii) secretion of the 68 proteolytic products for synaptic re-use. While mBDNF commonly activate TrkB, 69 astrocytic release of BDNFpro increases TrkB expression at the spines surface, which 70 captures sufficient neurotrophin signaling for LTP maintenance. Hence, the persistence in 71 synaptic strength is due to both an additional supply of mBDNF from astrocytic 72 microdomains and to an increase in the post-synaptic response to neurotrophin by the 73 prodomain. Astrocytic release of BDNFpro, ultimately provides the molecular basis for 74 retaining memory in the novel object recognition behavioral test. Thus, neurons and glia 75 are associated by neurotrophins in functional memory units, which build reinforcing 76 cellular and molecular loops enabling a persistent strengthening of the synapse and 77 memory consolidation. 
78 Results

79 proBDNF processing in astrocytes

80 Protoplasmic astrocytes are one type of morphologically characterized glial cells.

81 Cortical layer II/III astrocytes show a highly branched arborization and fine membrane

82 extensions in the cell periphery that enwraps synaptic contacts (16). We have previously

83 reported that cortical layer II/III astrocytes support clearing and recycling of proBDNF

$84(13,15,17)$ to sustain TrkB signaling and LTP maintenance in perirhinal cortex (15).

85 However, neither TrkB phosphorylation (18) nor the late-phase LTP $(19,20)$ are directly

86 regulated by proBDNF, suggesting that conversion of the inactive neurotrophin precursor

87 to an active product might play a more direct role. We now ask whether these same

88 astrocytes are proficient for direct cleavage of endocytic proBDNF following LTP-

89 inducing electrical stimulation.

90 Brain slices of control mice were prepared to examine the astrocytic origin of the

91 proBDNF processing. Their perirhinal cortex was previously injected in layer II/III with

92 adenoviral particles transducing green fluorescent protein (GFP) under the regulation of

93 glial fibrillary acidic protein (GFAP) promoter (AAV-GFAP-GFP) (21). Furthermore, in

94 order to avoid the injection procedure, transgenic mice stably expressing GFP under the

95 control of GFAP promoter (GFAP-GFP mice) (22) have been used. Slices were used for

96 field stimulation delivering $\theta$-burst-stimulation (TBS) evoking LTP (23). The occurrence

97 of proBDNF proteolytic processing was analyzed $10 \mathrm{~min}$ after stimulation by

98 immunohistochemistry using an antibody ( $\alpha$ BDNFpro) that specifically recognizes the

99 furin cleaved C-terminal end of the prodomain (Fig. 1a; (24)). This epitope is unavailable

100 in both intact proBDNF and mBDNF, providing that the antibody recognized the cleaved

101 BDNFpro, leaving undetected cleavage-resistant proBDNF (proBDNF ${ }^{\mathrm{CR}}$ ) and $\mathrm{mBDNF}$ in

102 the Western blot analysis (Fig. 1a). BDNFpro and GFP immunoreactivity was analyzed

103 by confocal microscopy to appreciate the specific distribution of the cleaved prodomain

104 in individual astrocytes (Fig. 1b and Supplementary Fig. 1a). GFP is a cytosolic protein

105 whose fluorescence defines the astrocyte in its entire cytoplasmic extension. This is a

106 feature that is ideal to achieve detection of BDNFpro in the astrocytic territorial volume.

107 Spatial overlap of BDNFpro and GFP was analyzed in a series of confocal stacks by 
using colocalization analysis of the two signals (Supplementary Fig. 1c). To facilitate BDNFpro visualization in the astrocytic territorial volume, BDNFpro/GFP colocalization was reconstructed in z-stacks. We observed sharp BDNFpro/GFP colocalization signal, as detected by $\alpha$ BDNFpro, in astrocytes from TBS-slices (Fig. 1c and Supplementary Fig. 1b). Cleaved prodomain detection was observed in small proportion at the cell body and in greater proportion in the cell periphery mostly matching with highly ramified processes. In marked contrast, basal stimulation induced little BDNFpro/GFP colocalization signal in astrocytes (Fig. 1c and Supplementary Fig. 1b). For quantification analysis, we used Mander's overlap and measured the extent of co-occurrence (25) between the two fluorophores. By this analytical parameter, the proportion of BDNFpro/GFP colocalization was increased in the whole cell (Fig. 1c and Supplementary Fig. 1b) or branches subcellular regions (Fig. 1c) in TBS vs. basal conditions. Additional colocalization parameter as the Pearson's correlation was used (Supplementary Fig. 1d), confirming that in TBS-slices BDNFpro/GFP colocalization signal significantly increased over baseline.

Glial cells were reported to lack de novo proBDNF synthesis under physiological conditions (26), suggesting that BDNFpro detection in these cells originates from the proBDNF that was previously endocytosed and then cleaved. Preventing proBDNF internalization in astrocytes would then prevent the precursor processing and accordingly BDNFpro detection. This assumption was validated by experiments conducted in loxPp75 ${ }^{\mathrm{NTR}}$-loxP mice (27) crossed with GLST-CreER ${ }^{\mathrm{T} 2}$ (28) and Rosa-CAGloxP-stoploxP(LSL)-R26R mice (29); for simplicity p75-flox mice (Supplementary Fig. 2a; (30)). Tamoxifen administration by intraperitoneal injection into adult p75-flox mice induced $\mathrm{p} 75^{\mathrm{NTR}}$ gene deletion, the carrier receptor for proBDNF uptake in these cells (15), and expression of $\beta$-Galactosidase ( $\beta$-Gal) reporter. Virtually, all $\beta$-Gal+ cells in the cortex showed the morphology of protoplasmic astrocytes (Supplementary Fig. $2 b$ ) and the vast majority of them were GFAP+ $(81 \pm 4 \%)$. Conversely, very few $\beta$-Gal+ cells expressed NeuN-neuronal marker $(1 \pm 0.7 \%)$, demonstrating that p75-flox mice allowed astrocytes selective targeting in the brain cortex in vivo. Immunoreactivity for BDNFpro was examined in slices from p75-flox mice 14 days post-tamoxifen (dptm). This latency 
ensured a significant depletion of $\mathrm{p} 75^{\mathrm{NTR}}$ protein following recombination in astrocytes

139 (Supplementary Fig. 2c). In p75 ${ }^{\mathrm{NTR}}$-deficient cells, BDNFpro/GFP signal was hardly 140 detectable in both TBS and basal stimulation (Fig. 1d). Thus, p75 ${ }^{\mathrm{NTR}}$-mediated proBDNF 141 endocytosis fed astrocytes with a cleavable pool of proBDNF.

142 Collectively, our data indicate that proBDNF is a substrate for proteolytic processing in 143 cortical layer II/III astrocytes following LTP-mediated internalization. Given that 144 endocytic proBDNF undergoes recycling in this potentiating condition (15), our data 145 suggest that astrocytes might convert proBDNF into BDNFpro and mBDNF at peri146 synaptic sites before routing to the secretory pathway.

\section{Localization of BDNFpro in astrocytic microdomains}

149 The intricate ramifications of astrocytes allow them to tightly enwrap the synaptic 150 terminals at organized peri-synaptic structures, the microdomains (31). Astrocytic microdomains can be distinguished into thick processes of micrometer scale $(\sim 10-15$

$152 \mu \mathrm{m}^{2}$ ) that host endoplasmic reticulum (ER) and mitochondria capable of generating 153 inositol-3-phosphate (IP3)-dependent $\mathrm{Ca}^{2+}$ signals and thin organelle-free structures of 154 submicron/nanometer-scale that fill the space between synapses (32-34). We speculated 155 that astrocytic proBDNF processing might be achieved on a rapid time scale at 156 microdomains and, possibly, within the same storage compartments orchestrating the 157 recycling process. Vesicular localization of the cleaved BDNFpro targeting astrocytic 158 microdomains has been evaluated to be in line with our hypothesis.

159 Subcellular localization of BDNFpro was initially resolved in super-resolution by using 160 structured illumination microscopy (SIM) (Fig. 2a). In TBS-slices from control mice, 161 BDNFpro/GFP colocalization signal appeared as a punctate pattern dispersed into cell 162 periphery of astrocytes. At higher magnification, BDNFpro/GFP colocalization was 163 present in membrane ramifications mostly shaped as finger-like extensions and flat 164 lamellar sheaths (Fig. 2a, b), which were recognized to be astrocytic structures contacting 165 the synapse. Quantification analysis confirmed that TBS induced a significant increase of 166 the colocalization signal $(45 \pm 6 \%$ of the total BDNFpro/GFP puncta detected in 167 astrocytes) in these specific structures. Given the nanometer scale resolution of the SIM 
super-resolution microscopy (lateral res. $115 \mathrm{~nm}$; axial res. $250 \mathrm{~nm}$ ), our data suggest for an enrichment of the cleaved prodomain in high membranous elaborations of the cell periphery viewing the dimensions and typical morphology of microdomains.

To firmly assess this conclusion, we extended our investigation at ultra-structural levels (15). Ultra-thin sections from TBS-slices were examined by transmission electron microscopy (EM) in pre-embedding experiments (Fig. 3a). At 70,000-100,000-fold magnification, immunogold labeling was observed using $\alpha$ BDNFpro antibody ( $\alpha$ BDNFpro-gold) in (i) vesicular structures at pre-synaptic terminals typically displaying clouds of synaptic vesicles opposed to post-synaptic density structures (Fig. 3b); (ii) postsynaptic terminals (Fig. 3b); and (iii) astrocytes giving rise to fine astrocytic processes in close proximity to the synapses (Fig. 3c). Consistent with our data that BDNFpro in astrocytes is generated from endocytic proBDNF processing in response to neuronal activity, TBS-slices showed a higher number of $\alpha$ BDNFpro-gold grains in astrocytes filling the space between synapses $(38 \pm 7 \%$ of the total gold particles detected in astrocytes) with respect to non-stimulated slices $(12 \pm 4 \%$ of the total gold particles detected in astrocytes). Most gold particles were organized in groups of many grains and were concentrated peri-synaptically $(46 \pm 4 \%$ of the total gold particles detected in astrocytes) in a membrane-delimited area of $230 \mathrm{~nm}$ radius surrounding synaptic contacts (Supplementary Fig. 3a). This distance represents the maximum of the astrocyte volume fraction when astrocytes cover dendritic spines and axonal boutons (35). A similar enrichment was previously reported (15), using amBDNF-gold (Supplementary Fig. 3b) or aproBDNF-gold (Supplementary Fig. 3c) potentially detecting both proBDNF and mBDNF isoforms or proBDNF and BDNFpro, respectively, suggesting for proBDNF processing in this area.

Lastly, we addressed the vesicular localization of BDNFpro. Endocytic proBDNF was shown to localize in vesicles expressing both $\mathrm{p} 75^{\mathrm{NTR}}(13,15)$ and Vamp2 $(36)$, a component of the core SNARE complex; thus indicating that intracellular storage of endocytic proBDNF in astrocytes involves vesicles equipped with the molecular machinery deputed to both endocytosis and exocytic fusion (recycling vesicles). In line with these findings, we discovered BDNFpro/GFP colocalization that overlapped with 
198 Vamp2/GFP colocalization in the astrocytic territory (Fig. 4a), suggesting that the 199 processing of the precursor takes place at recycling vesicles. Moreover, the vesicular 200 localization of the prodomain in astrocytes was analyzed by BDNFpro/Vamp2 201 colocalization using SIM super-resolution microscopy, thereby at a resolution limit 202 compatible with the dimension of astrocytic microdomains. In TBS-slices, 203 BDNFpro/Vamp2 colocalization signal appeared as a punctate pattern reminiscent of 204 vesicular structures dispersed into cell periphery of astrocytes (Fig. 4b). Vesicular 205 localization of BDNFpro was further observed at ultrastructural level (Fig. 4c, d); 206 vesicles filled with BDNFpro-gold were occasionally seen at the astrocytic limiting 207 membrane near synaptic endings. Similar vesicular localization was previously reported 208 using aproBDNF-gold or $\alpha \mathrm{mBDNF}$-gold (15); however, a reliable quantification of these 209 storage organelles was inhibited by our pre-embedding procedure (15, 37). This 210 procedure resulted to a poor preservation of ultrastructural details in astrocytes, while 211 ensuring the specificity of BDNFpro signal by preserving the antibody antigenicity. We

212 conclude that vesicles containing BDNFpro are created at astrocytic microdomains in 213 response to TBS.

\section{Astrocytic BDNFpro is sufficient for LTP maintenance}

216 A central hypothesis arising from proBDNF processing in recycling vesicles is that the 217 resulting end products participate in gliotransmission, supplying astrocytes with a 218 releasable source of active neurotrophins and enabling LTP maintenance. Conversion of 219 proBDNF to mBDNF would typically satisfy this requirement. However, given that 220 BDNFpro and mBDNF (15) share similar vesicular localization, we formulated the 221 original hypothesis that BDNFpro individually participates to synaptic strengthening via 222 the recycling process.

223 To assess the contribution of BDNFpro in LTP, we performed LTP-rescue experiments in 224 conditional p75-flox mice as reported previously (15). In these experiments astrocytes are 225 incapable of proBDNF uptake, resulting in a short-lived potentiation that declined to 226 baseline about $140 \mathrm{~min}$ after TBS (Supplementary Fig. 4a). Conversely, expression of 227 proBDNF (Supplementary Fig. 4b), but not cleavage resistant proBDNF ${ }^{\mathrm{CR}}$ 
(Supplementary Fig. 4c) in astrocytes restores LTP for the $180 \mathrm{~min}$ duration of the recording to levels exhibited by control littermates. An ectopic source of cleavable proBDNF in $\mathrm{p} 75^{\mathrm{NTR}}$-deficient astrocytes would then be assumed to replenish endocytic proBDNF and compensate for the lack of recycling in these cells, restoring the LTP deficits. Using this strategy, we expressed BDNFpro specifically in $\mathrm{p} 75^{\mathrm{NTR}}$-deficient astrocytes for LTP assessment.

First, we engineered a lentiviral construct $\left(\mathrm{LV}-\mathrm{BDNFpro}^{\text {stop }}\right)$ for BDNFpro expression (Supplementary Fig. 5a). In this construct, a loxP-GFP-STOP-loxP cassette allows GFP expression while preventing for BDNFpro expression. The GFP-STOP cassette is removed in the presence of Cre recombinase, finally resulting in GFP loss and activation of the BDNFpro transgene and Tomato reporter. We injected LV-BDNFpro ${ }^{\text {stop }}$ into perirhinal cortices of p75-flox mice at 0 or $12 \mathrm{dptm}$. At $14 \mathrm{dptm}$ (Supplementary Fig. 5b), cortical slices were prepared and stained for the astrocytic marker GFAP, the mouse reporter $\beta$-Gal and the viral reporter Tomato. Most Tomato+ cells $(98 \pm 4 \%)$ were recombined astrocytes (GFAP $+/ \beta-\mathrm{Gal}+)$ indicating the specificity of the lentiviral construct. In addition, we found that transduced astrocytes from both injection times expressed comparable levels of BDNFpro immunoreactivity, which was in the range of the one observed in TBS-slices from control littermates (Supplementary Fig. 5c). This is consistent with the expectation that virally transduced BDNFpro replaces the prodomain generated by endocytic proBDNF processing.

In parallel, slices were used for field recordings. While basal synaptic transmission (input-output; Supplementary Fig. 5d) and synaptic facilitation (paired-pulse facilitation; Supplementary Fig. 5e) were unaffected by LV-BDNFpro ${ }^{\text {stop }}$ transduction, slices from p75-flox mice injected with the virus showed to restore long-lasting LTP deficits (Fig. 5a and Supplementary Fig. 4d). Thus, supplying astrocytes with the sole BDNFpro expression compensates for the physiological presence of proBDNF uptake, processing, and final recycling of the proteolytic products. Prerequisite for this process is that virally transduced BDNFpro in astrocytes undergoes to secretion enabling LTP maintenance. To assess this issue, we designed a lentiviral construct for specific expression of Tetanus Toxin (TeTN) light-chain (LV-TeTN ${ }^{\text {stop }}$ ) in astrocytes (Supplementary Fig. 5a). 
258 Sustained astrocytic expression of TeTN - a protease known to cleave the SNARE 259 protein Vamp2 (36) - is expected to inhibit the fusion of all secretory vesicles including 260 those containing the neurotrophin (13). We injected LV-TeTN ${ }^{\text {stop }}$ together with LV261 BDNFpro $^{\text {stop }}$ in perirhinal cortices of p75-flox mice the last day of tamoxifen treatment 262 (Fig. 5b). At $14 \mathrm{dptm}$, cortical slices were used for LTP recordings. Slices from double263 injected mice showed LTP that decayed to baseline 120-140 min in response to TBS; 264 while co-injecting LV-GFP ${ }^{\text {stop }}$ and LV-BDNFpro ${ }^{\text {stop }}$ viruses into the contralateral 265 hemisphere of the same mice showed to restore the LTP deficits to the levels exhibited by 266 control littermates. Thus, docking and fusion of Vamp2-secretory vesicles containing 267 BDNFpro are required for LTP maintenance.

268 Whether BDNFpro is sufficient for rescuing LTP deficit was next investigated. Slices 269 from p75-flox mice were perfused with exogenous BDNFpro (10 ng/ml) for $10 \mathrm{~min}$ (Fig. 270 5c). This timing was chosen as it correlates with the duration of proBDNF recycling in 271 this cortical area (15). Exogenous application of the BDNFpro fragment initiated 2 min 272 before TBS and was maintained for additional $8 \mathrm{~min}$. We found that fEPSP was 273 significantly restored in p75-flox mice receiving recombinant BDNFpro compared to 274 vehicle-treated slices. Given that exogenous administration of mBDNF (10 ng/ml), but 275 not cleavage-resistant proBDNF ${ }^{\mathrm{CR}}(20 \mathrm{ng} / \mathrm{ml})$, similarly rescued LTP deficits 276 (Supplementary Fig. 6a), our data indicate that proBDNF processing provides a 277 mechanistic link between proBDNF clearing and subsequent recycling of the converted 278 products, both possessing same individual ability to sustain LTP. Moreover, when TBS 279 was omitted from the paradigm, BDNFpro application had no effect on fEPSP responses 280 over a 180 min period of baseline test-stimulation (Supplementary Fig. 6b), indicating 281 that BDNFpro requires TBS for participating in synaptic strengthening. The rescuing 282 effect of recombinant BDNFpro is a transient phenomenon that saturates over time, as 283 demonstrated by applying BDNFpro for $10 \mathrm{~min}$ at different time points after TBS 284 (Supplementary Fig. 6c). The period of BDNFpro dependency ended about 110 min from 285 TBS; after this time, application of the pro-fragment could no longer restore LTP deficit. 286 Thus, BDNFpro is required for a limited time window to sustain LTP. 
287 Collectively, our data indicate astrocytes to be as proficient for BDNFpro release, which

288 fulfills the function to mediate the switch from early- to late-phase LTP.

290 BDNFproval/Met preserves bioactivity

291 A single-nucleotide polymorphism in the human BDNF gene results in valine (Val) to 292 methionine (Met) substitution at codon 66 (Val66Met) in the prodomain region causing 293 memory alteration in humans $(38,39)$ and impaired synaptic strengthening in transgenic 294 mice carrying the mutation (40). By these premises, we investigated whether structural 295 changes induced by this amino acid substitution might compromise BDNFpro function 296 on LTP maintenance. Slices from p75-flox mice were perfused with recombinant 297 BDNFpro carrying the genetic variation (BDNFproval66Met) and subjected to TBS. We 298 found that BDNFpro ${ }^{\text {Val66Met }}(10 \mathrm{ng} / \mathrm{ml})$ applied for $10 \mathrm{~min}$ in stimulated slices (Fig. 5d) 299 retained the ability to restore LTP deficit. Thus, Val66Met substitution does not subtract 300 bioactivity to BDNFpro in our experimental context.

\section{Astrocytic BDNFpro increases post-synaptic TrkB signaling}

304

305

306

307

BDNFpro is believed to be ligand for the sortilin family member receptor SorCS2 (41). A potential function attributed to SorCS2 is to act as co-receptor for TrkB, assisting the assembling and targeting of TrkB/SorCS2 complex to post-synaptic membranes (42). In this way, it recruits sufficient TrkB signaling for LTP maintenance. Once recycled by astrocytic microdomains, BDNFpro would then support aggregation and targeting of TrkB/SorCS2 complex at post-synaptic sites.

To assess this mechanistic issue, cultured cortical neurons expressing GFP were subjected to in situ proximity ligation assay (PLA) (42). This experimental strategy requires PCR-amplification to hybridize fluorescent DNA probes coupled to secondary antibodies (arabbit IgG and agoat IgG) targeting $\alpha \operatorname{TrkB}$ (rabbit) and $\alpha$ SorCS2 (goat) primary antibodies (Fig. 6a). Hybridization takes place only when probes are localized less than $40 \mathrm{~nm}$ apart, which reflects TrkB/SorCS2 clustering in our experimental context. In vehicle-treated neurons, basal PLA ${ }^{\text {TrkB/SorCS2 }}$-signal appeared as sparse fluorescent pattern (Fig. 6b). Fluorescent signal increased after treating neurons with 
317 exogenous BDNFpro $(10 \mathrm{ng} / \mathrm{ml})$ for $10 \mathrm{~min}$. PLA ${ }^{\mathrm{TrkB} / \mathrm{SorCS} 2}$-signal was prominent in the 318 plasma membrane of both cell body and dendritic processes, indicating a clear post319 synaptic localization of TrkB/SorCS2 aggregates. Strikingly, PLA ${ }^{\text {TrkB/SorCS2 }}$-signal in 320 dendrites was predominately concentrated in proximity of spines, appearing as membrane 321 protrusion extending from dendritic processes (Fig. 6b). Quantitative analysis confirmed 322 the increase of PLA ${ }^{\text {TrkB/SorCS2}}$-signal in BDNFpro-treated vs. vehicle-treated cultures (Fig. 323 6c). The specificity of BDNFpro treatment was assessed by pre-treating neurons for 20 324 min with $\alpha$ SorCS2 $(20 \mu \mathrm{g} / \mathrm{ml}$ ) (Fig. 6b), a blocking antibody that is known to prevent 325 TrkB/SorCS2 complex formation (42). In the presence of aSorCS2 pre-treatment, 326 BDNFpro fragment could no more exert its aggregating effect and PLA ${ }^{\text {TrkB/SorCS2 }}$-signal 327 could not be seen (Fig. 6c). Moreover, when neurons were treated with recombinant $328 \mathrm{mBDNF}(10 \mathrm{ng} / \mathrm{ml})$, or cleavage-resistant proBDNF ${ }^{\mathrm{CR}}(20 \mathrm{ng} / \mathrm{ml}), \mathrm{PLA}^{\mathrm{TrkB} / \mathrm{SorCS} 2}$-signal 329 had not changed significantly as compared to vehicle-treated neurons (Fig. 6c); that 330 indicates that, within BDNF isoforms, TrkB/SorCS2 aggregation is a unique function of 331 BDNFpro. PLA analysis was extended to cortical slices from control mice 10 min after 332 basal- or TBS-stimulation (Fig. 6d). We measured in a quantitative analysis, $333 \mathrm{PLA}^{\mathrm{TrkB} / \mathrm{SorCS} 2}$ signal that colocalized with the postsynaptic marker NeuN 334 (NeuN/PLA ${ }^{\text {TrkB/SorCS2 }}$ ) for cell body localization, and PSD95 (PSD95/PLA ${ }^{\text {TrkB/SorCS2}}$ ) for 335 spines localization at the stimulated areas. In TBS-slices, both NeuN/PLA ${ }^{\text {TrkB/SorCS2 }}$ and 336 PSD95/PLA ${ }^{\text {TrkB/SorCS2 }}$ colocalizations had significantly increased with respect to baseline 337 stimulation, indicating that TrkB and SorCS2 aggregate at post-synaptic sites in response 338 to potentiating conditions. The astrocytic dependency on TrkB/SorCS2 aggregation was 339 next examined in TBS-slices from p75-flox mice previously injected with control LV340 GFP $^{\text {stop }}$ or LV-BDNFpro ${ }^{\text {stop }}$ viruses. TBS-slices transduced with LV-BDNFpro ${ }^{\text {stop }}$ showed 341 higher levels of NeuN/PLA ${ }^{\text {TrkB/SorCS2 }}$ signals with respect to slices transduced with LV342 GFP $^{\text {stop }}$ (Fig. 7a). Thus, BDNFpro sourced by astrocytes increases TrkB/SorCS2 343 aggregation at post-synaptic sites following LTP-inducing neuronal stimulation.

344 Finally, high-localized TrkB/SorCS2 aggregation would result in increased TrkB activity. 345 To assess this possibility, we examined TrkB phosphorylation (pTrkB) by using $\alpha$ 346 phospho-TrkB (Tyr816) antibody ( $\alpha$ TrkB). Given that pTrkB is essentially a fraction of 
the total TrkB levels, we measured, in a quantitative analysis, pTrkB that colocalizes with TrkB immunoreactivity. We found that the levels of pTrkB/TrkB colocalization had significantly increased in TBS-slices compared to non-stimulated slices from p75-flox mice injected with LV-BDNFpro ${ }^{\text {stop }}$ or TBS-stimulated slices from control littermates injected with LV-GFP ${ }^{\text {stop }}$ (Fig. 7b and Supplementary Fig. 7). Moreover, this increase is comparable to the one observed in TBS vs. baseline condition in control littermates (Fig. 7b). Thus, astrocytic BDNFpro recruits TrkB expression on adjacent spines for tight temporal, spatial and stimulus- dependent TrkB phosphorylation.

\section{Astrocytic BDNFpro restores memory retention}

Substantial evidence suggests that the perirhinal cortex plays a critical role in familiaritybased object recognition (ORT) memory $(43,44)$.

To assess whether BDNFpro originated by astrocytes is involved in recognition memory in vivo, we injected LV-GFPstop or LV-BDNFpro $^{\text {stop }}$ viruses in perirhinal cortex of p75flox mice the last day of tamoxifen treatment and performed the ORT $14 \mathrm{dptm}$. Mice performed the ORT with a retention interval between the sample phase and the test phase of $10 \mathrm{~min}$ and $24 \mathrm{~h}$ (Fig. 8a). The discrimination index (DI) can vary between +1 and -1 , where positive scores indicate more time spent with the novel object and a zero or negative score indicates equal or less time spent with the novel object. In the DI (Fig. 8b) p75-flox mice injected with control virus showed positive values at 10 min time that decreased to almost zero at $24 \mathrm{~h}$. Thus, when astrocytic proBDNF uptake was blocked, as in p75-flox mice, memory consolidation was prevented and mice showed a significant memory deficit in the ORT (15). Strikingly, p75-flox mice injected with a virus transducing BDNFpro showed similar positive value at both $10 \mathrm{~min}$ and $24 \mathrm{~h}$ times, as for control littermates, indicating that astrocytic BDNFpro is sufficient for the consolidation of this type of memory. Exploration time at the sample phase was comparable between groups (Fig. 8c). Thus, BDNFpro in astrocytes could reverse the memory defect exhibited by p75-flox mice, demonstrating its necessity to memory consolidation in vivo. 


\section{Discussion}

376 At the micro/nanoscale, neural plasticity emerges as changes in the spatiotemporal 377 pattern of activation of different synaptic components. While much attention has been 378 given to pre- and post-synaptic sites for coordinating long-lasting synaptic strengthening $379(6,11)$, research in this regard has long time ignored the function of astrocytic 380 microdomains. The close anatomical interface between pre- and post-synaptic neurons 381 and astrocytes has been referred to as the "synaptic triate" (45) or "tri-partite synapse" 382 (46) in which astrocytic microdomains are proposed as regulatory units of neuron-glia 383 interaction. Our work indicates these cellular structures to be specialized in prolonging 384 synaptic potentiation by clearing and converting the inactive proBDNF in functional 385 BDNFpro and mBDNF products, thereby increasing temporal, spatial and stimulus386 dependent neurotrophin availability at spines. BDNFpro and mBDNF reinforce TrkB 387 signaling via adaptive molecular mechanisms that promote LTP maintenance and

400

401

402

403

404 memory consolidation. This molecular functionalization process might involve individual or small groups of synapses covered by thin (nanometer scale) or thick $\left(\sim 10-15 \mu \mathrm{m}^{2}\right)$ microdomains, respectively $(31,47,48)$. It is worthy of note that the dimensions of thick microdomains coincide with the reported maximum distance between functionally related spines (49). Studies on neurons in the visual cortex have recently reported that dendritic spines, within less than $\pm 10 \mu \mathrm{m}^{2}$ distance, tend to share receptive field properties, while more distant spines are functionally uncorrelated (50). It is therefore reasonable to assume that the spatial extent of BDNFpro localization at microdomains might be a constraining factor for the effect of these substructures in synaptic modifications. This spatially restricted mechanism of plasticity may finally participate in long-term memory engram formation (51) and memory capacity (52).

\section{Confinement of a molecular memory in astrocytic microdomains}

Astrocytic proBDNF is inactive in the precursor state and can be converted to active mBDNF at microdomains. This inactive-to-active transition increases mBDNF availability exceeding the transient, activity-dependent neurotrophin secretion from neurons. This represents per se a positive cellular and molecular loop (proBDNF transfer 
from neurons to astrocytes $==>$ processing and storage of the precursor $==>\mathrm{mBDNF}$ transfer from astrocytes to neuron) that survives to LTP induction. However, our data

407 provide further significance to this molecular activation; not only mBDNF, but also the 408 BDNFpro turned out to possess an independent function. Once generated, this byproduct 409 participates in astrocytic recycling by inducing changes in TrkB levels (Fig. 7a) as well 410 as in this receptor phosphorylation at post-synaptic sites (Fig. 7b). Thus, BDNFpro 411 operates synaptic adaptations once LTP is expressed that are relevant for its later 412 stabilization (Fig. 5a, c). Mechanistically, BDNFpro acts as an activator of TrkB/SorCS2 413 aggregation by spine targeting (Fig. 6b-d). This is a new physiological role attributed to 414 the cleaved BDNFpro, which on behalf of its structural instability has been recently 415 assigned to co-secretion with $\operatorname{mBDNF}(37,41)$ and assumed to possess independent 416 physiological functions $(24,53)$. While previous data reported that exogenous application 417 of proBDNF (12) or BDNFpro (53) enhances long-term depression (LTD)-inducing low 418 frequency-stimulation in the hippocampus, we provide compelling evidence that in 419 perirhinal cortex astrocytic proBDNF recycling and therefore astrocytic release of its 420 proteolytic products BDNFpro and mBDNF do not participate in this form of facilitation 421 (15). This emphasizes the relevance to consider different brain regions and to identify the 422 precise location of neurotrophin secretion at synaptic level. Although extremely 423 challenging experimentally, these important issues must be addressed in order to clarify 424 current inconsistency of the involvement of neurotrophins in synaptic plasticity and 425 processes related to memory (54).

426 Overall, the main question underlying astrocytic proBDNF processing can be further 427 detailed: is the persistence in synaptic strength due to an additional supply of active $428 \mathrm{mBDNF}$ from astrocytic microdomains or is it due to an increase in the post-synaptic 429 response to this neurotrophin by the prodomain? We suggest cooperation between 430 BDNFpro and mBDNF for convergent, but independent post-synaptic signaling. On the 431 one hand, exogenous administration of mBDNF could restore LTP deficit in p75-flox 432 mice (15), implicitly assuming that mBDNF supplied by astrocytes is sufficient for LTP 433 maintenance. On the other hand, astrocytic expression of the sole BDNFpro in p75-flox 434 slices (Fig. 5a) or its exogenous administration (Fig. 5c) equally rescued the LTP deficits. 
435 This suggests a mechanism, in which the basal levels of $\operatorname{TrkB}$ at spines cannot recruit 436 sufficient mBDNF signaling for LTP maintenance. On recycling, BDNFpro increases 437 TrkB levels at post-synaptic sites, allowing the receptor to act as a central controller. 438 Accordingly, BDNF-TrkB signaling induces molecular adaptation underlining long439 lasting synaptic strengthening (11) and shapes structural plasticity (55) via both TrkB 440 tyrosine (56) and serine/threonine phosphorylation (57). While this essential regulation 441 saturates over time, the rescuing effect on LTP deficits by BDNFpro persists for the 442 entire duration of the early-phase LTP expression, ending up at the initiation of the late443 phase potentiation (Supplementary Fig. 6c). In marked contrast, mBDNF is only required 444 for the first 10-20 min after stimulation, which is the predicted time of astrocytic 445 proBDNF recycling (15). Thus, prodomain and mature BDNF integrate various signaling 446 pathways once LTP is expressed, and the conversion of the precursor acts as an inducible 447 "molecular switch". Therefore, proteolytic cleavage of pro-neurotrophins is an important 448 regulatory step for the direction of neurotrophin function. In conclusion, proBDNF 449 processing does not fully accomplish the classical model of self-sustained molecular 450 alteration (1); instead, it offers a switching mechanism from one inactive state to multi451 functional one. This specifies strengthening mechanism for a persistent post-synaptic 452 signaling, as it is required for a "molecular memory" to maintain LTP.

453 Spine targeting of TrkB tackles the underlying changes that occur at the synapse once 454 LTP is expressed, reinforcing the common knowledge that LTP maintenance take place 455 post- synaptically (6). However, our data also provide evidence that the molecular basis 456 responsible for these changes can be confined elsewhere. To understand the molecular 457 foundation of LTP maintenance, it is therefore essential to know from which site of the 458 synapse the "molecular memory" is originated. Since the discovery of LTP, most studies 459 focused on whether pre- and/or post-synaptic sites are the compartments confining 460 molecular modifications relevant for LTP maintenance; here we moved away from 461 neurons and demonstrated that astrocytic microdomains are a central player for memory 462 storage. Focus on the site of expression of BDNFpro was made possible by using electron 463 microscopy (Fig. 3). BDNFpro-gold particles were seen at pre- and post-synaptic 464 terminals and astrocytic microdomains closely interacting with these synaptic complexes. 
465 Gold particles residing in these synaptic sites account for highly intricate trafficking 466 events induced by TBS: BDNFpro could be (i) synthesized at pre- and/or post-synaptic 467 sites; (ii) transported to pre- and/or post-synaptic sites; (iii) internalized in pre- and/or 468 post-synaptic compartments as well as in astrocytic microdomains. Given this 469 complexity, it is of note that BDNFpro localization at astrocytic microdomains is the only 470 relevant localization required for LTP maintenance. Although EM analysis showed a 471 variety of synapses in terms of shapes and sizes, the one thing that was constant was the 472 enrichment of BDNFpro-gold particle at peri-synaptic astrocytes (Fig. 3). This suggests 473 the general occurrence of proteolytic conversion in this specialized area. Moreover, 474 BDNFpro colocalized with the SNARE protein Vamp2 (Fig. 4 a, b) and intoxication with 475 TeTN - a protease known to cleave Vamp2 - inhibited BDNFpro secretion (Fig. 5b). This 476 indicates that astrocytic vesicles store the processed neurotrophins at a place that is 477 functional to synaptic use. Thus, astrocytic vesicles could serve as a reservoir of 478 functional neurotrophin products that are available for synaptic needs. Such a modified 479 and stable enrichment of astrocytic microdomains could serve as signaling hub of 480 potentiated synapses providing an appealing model for information storage.

\section{Astrocytic microdomains regulate BDNF specificity}

483 The observation that persistent LTP and its associated synaptic changes are synapse484 specific (58) raises the issue of how BDNF availability can be independently regulated in 485 individual synapses. If BDNF could leak from a stimulated synapse, it would then 486 massively, but incorrectly activate many additional synapses in spite of an appropriate 487 stimulation. The idea of "synaptic capture" appears to be a valuable model in this context 488 (58, 59). This implies that newly synthesized BDNF by neurons is delivered to all 489 synapses, but it is only used at synapses that have been tagged by activity. In accord to 490 this model, TrkB, the correlated molecular tag for mBDNF (60), could capture mBDNF 491 at selected synapses. Our finding that astrocytic BDNFpro increases TrkB/SorCS2 492 aggregation at the spine surface (Fig. 6b and Fig. 7a), supports the idea that proBDNF 493 processing in microdomains may cooperate to selectively tag activated synapses. This 494 specific process will then provide the capture of $\mathrm{mBDNF}$ for enhancing TrkB 
phosphorylation (Fig. 7b). Direct demonstration of the astrocytic origin of TrkB tagging 496 would need to perform a two-pathway experiment (60) in perirhinal cortex of p75-flox 497 mice; a brain area not permissive for this type of recording. However, this experiment 498 conducted in hippocampus (42) confirmed that SorCS2 binds TrkB to facilitate 499 TrkB/SorCS2 translocation at posts-synaptic sites for both synaptic tagging and LTP 500 maintenance. Overall, our data suggest that astrocytic BDNFpro triggers mBDNF capture 501 via TrkB tagging; thereby increasing selectivity and responsiveness to the neurotrophin at 502 potentiated synapses.

\section{Linking LTP maintenance to memory consolidation}

The proposed critical role and mode of action of astrocytic proBDNF processing for LTP maintenance can be extended to long-lasting memory. Specifically, is proBDNF conversion important for memory consolidation? And as a consequence of this, is BDNFpro central to this type of memory? We demonstrated that viral expression of BDNFpro in perirhinal cortex of p75- flox mice resulted in mice that spent more time exploring a novel object than a familiar one during the 24 h-test phase (Fig. 8b). Astrocytic release of this "byproduct" is then correlated to long-lasting memory of the task. This finding supports the idea that astrocytic microdomains participate to changes in the strength of neuronal connections contributing to the most attractive cellular model for learning and memory, first defined by Hebb in 1949 (61). His hypothesis that synaptic strengthening for learning and memory occurs as a consequence of coincident activity between pre- and post-synaptic compartment now requires the integration of a new player, the astrocytic microdomains. This cyto-architectural structure accounts on functionally isolated subcellular domains that facilitate local homeostasis by redistributing ions, removing neurotransmitters, and releasing factors to influence moment-to-moment synaptic activity $(8,62)$. Our identification of an astrocytic "molecular memory" indicates that microdomains are also capable of sensing and integrating signals for persistent synaptic strengthening that are involved in memory consolidation in the brain. There is now overall agreement that persistent modification of the synaptic strength, via sustained LTP, represents a primary mechanism for the 
525 formation of memory engrams (63). The contribution of astrocytes to synaptic engrams 526 has been largely ignored in the past $(9,64,65)$; however, it is evident that astrocytes 527 appear to be an important cellular interface to control and modify neuronal data 528 processing by their close physical contact with synapses (66). Astrocytic microdomains 529 could then provide local support over synaptic potentiation and the global control of 530 neuronal ensembles engaged in memory circuits. Thus, cortical astrocytes possess unique 531 features that ideally position them for locally responding to synaptic demand and to sense 532 the surrounding neuronal network activity. In this way, they can operate integrative 533 functions for learning and memory. 
534 Methods

535 Experimental models

536 All experiments were performed in accordance with European Union guidelines as 537 approved by the institutional animal care and utilization committee (authorizations $538 \mathrm{n}^{\circ}$ 507/2017-PR; 76/2020 PR). p75-flox mice were generated by crossing loxP-p75 ${ }^{\mathrm{NTR}}$. 539 loxP mice kindly provided by B. (University of Michigan School of Dentistry, USA) with 540 GLAST-CreER ${ }^{\mathrm{T} 2}$ Rosa-CAGloxP-stop-loxP(LSL)-R26R mice kindly provided by Prof. 541 M Gotz (LMU, Munich, Germany). GFAP-GFP mice were kindly provided by Prof. A. 542 Buffo (NICO, Torino, Italy). Animals were housed in a $12 \mathrm{~h} \mathrm{light/dark}$ cycle with 543 unrestricted access to food and water. For timed pregnancies, the plug date was 544 designated as E0 and the date of birth was defined as P0.

\section{Tamoxifen treatment}

547 Male mice from p75-flox and control littermates were treated with $1 \mathrm{mg}$ of tamoxifen 548 (Sigma Aldrich, Cat\#T5648) dissolved in corn oil twice a day for 5 consecutive days at 549 age between P35 and P50.

\section{Stereotaxic surgery}

552 For virus delivery, 0 or 12 days after the last day of tamoxifen administration, mice were 553 deeply anesthetized and viral particle ( $1 \mu 1$ in volume) was infused into perirhinal cortex 554 from each hemisphere (coordinates from Bregma: anteroposterior - $2 \mathrm{~mm}$, lateral $\pm 4,2$ $555 \mathrm{~mm}$, ventral $+2,8 \mathrm{~mm}$ ). Viral delivery was obtained through the insertion of capillary 556 glasses (WPI) connected to a manual syringe pump (Narishige). Mice were allowed to 557 recover and housed in standard cages until the day of sacrifice.

559 Viral vectors

560 pAAV.GFA104.PI.eGFP.WPRE.bGH (AAV-GFAP-GFP) adenoviral vector was used to 561 express GFP in astrocytes specifically. This vector was a gift from Philip Haydon 562 (Addgene viral prep \# 100896-AAV5; http://n2t.net/addgene:100896; RRID: 563 Addgene_100896). 
Lentiviral vectors were used for Cre-dependent expression of BDNFpro (LVBDNFpro $^{\text {stop }}$ ), proBDNF (LV-proBDNF $\left.{ }^{\text {stop }}\right)$, proBDNF$^{\mathrm{CR}}$ (LV-proBDNF $\left.{ }^{\mathrm{CR} s t o p}\right)$ and light-

566 chain of Tetanus toxin $(\mathrm{TeTN})\left(\mathrm{LV}-\mathrm{TeTN}{ }^{\text {stop }}\right)$. Lentiviral vectors were produced starting 567 from pLV which drives gene expression under the cytomegalovirus (CMV) promoter. A 568 gene-cassette including GFP encoding sequence was flanked by two modified loxP sites 569 (lox2272). This prevented the formation of unwanted ATG start codons after Cre570 mediated recombination. The GFP expression was stopped by two stop codons. Next, a 571 single EcoRV-blunt cloning site, an IRES2 (internal ribosomal entry site2) followed by 572 TandemTomato (Tomato) was cloned in 5' direction before the WPRE lentiviral vector 573 element. This construct showed strong GFP expression and weak expression of IRES2574 Tomato. Under Cre-mediated recombination, the GFP was lost and the IRES2-Tomato 575 cassette was under control of the promoter. Next, the EcoRV-blunt site was used to 576 introduce the coding sequence of BDNFpro, proBDNF, proBDNF ${ }^{\mathrm{CR}}$ and light-chain of 577 Tetanus toxin (TeTN). Lentiviral particles were produced in HEK293T cells. Lentiviral 578 expression vectors were co- transfected with the pseudotyping vector pMD2.G and the 579 packaging vector pCMVR8.91. Lentiviral particles were separated from the supernatant 580 by ultracentrifugation and stored at $-80^{\circ} \mathrm{C}$ in $50 \mathrm{mM}$ Tris- $\mathrm{HCl}, \mathrm{pH} 7.4,130 \mathrm{mM} \mathrm{NaCl}, 10$ $581 \mathrm{mM} \mathrm{KCl}, 5 \mathrm{mM} \mathrm{MgCl}$.

\section{Electrophysiological recording}

584 Slices from perirhinal cortex were prepared from p75-flox, control littermates or GFAP585 GFP mice. The brain was removed and placed in cold oxygenated $\left(95 \% \mathrm{O}_{2}\right.$ and $\left.5 \% \mathrm{CO}_{2}\right)$ 586 artificial cerebrospinal fluid (ACSF) containing $124.0 \mathrm{mM} \mathrm{NaCl}, 4.4 \mathrm{mM} \mathrm{KCl}, 1 \mathrm{mM}$ $\mathrm{NaH}_{2} \mathrm{PO}_{4}, 2.5 \mathrm{mM} \mathrm{CaCl} 2,1.3 \mathrm{mM} \mathrm{MgCl} 2,26.2 \mathrm{mM} \mathrm{NaHCO} 3,10 \mathrm{mM}$ glucose, and 2 mM L-ascorbic acid. Horizontal cortex slices (300 $\mu \mathrm{m}$ thick) were prepared using a vibratome and maintained in a chamber containing oxygenated ACSF at room temperature. After a minimum recovery period of $1 \mathrm{~h}$, a single slice was transferred into a submersion recording chamber perfused $(3 \mathrm{ml} / \mathrm{min})$ with oxygenated $\mathrm{ACSF}$ at $32^{\circ} \mathrm{C} \pm$ 593 stimulus generator (WPI, stimulus isolator A360) connected through a stimulus isolation 
594 unit to a concentric bipolar electrode (40-80 KU, FHC) positioned in layers II/III on the 595 temporal side of the rhinal sulcus. Evoked extracellular fEPSPs were recorded using an 596 Axoclamp-2B amplifier (Axon Instruments) with ACSF-filled glass micropipette pulled 597 on a vertical puller (Narishige PC-10, resistance [ $<5 \mathrm{MU}]$ ), inserted in layers II/III at 598 around $500 \mathrm{~mm}$ from the stimulation electrode, and analyzed using Axoscope 8.0 599 software. Baseline responses were obtained every $30 \mathrm{~s}$ with a stimulus intensity adjusted 600 to induce $50 \%$ of the maximal synaptic response. After 20 min of stable baseline, LTP 601 was evoked by TBS (100 Hz; four sets of stimulations delivered $15 \mathrm{~s}$ apart, each one 602 consisting of ten bursts of five pulses at $100 \mathrm{~Hz}$ with inter-burst intervals of $150 \mathrm{~ms}$ ). 603 fEPSPs were plotted as amplitude. Each point represents the responses every $30 \mathrm{~s}$ 604 expressed as means \pm SEM.

605 Input/output curves were produced delivering stimuli ( $0.1 \mathrm{~ms}$ duration) to the stimulation 606 electrode with stimulation intensities from 50 to $400 \mu \mathrm{A}$ in steps of $50 \mu \mathrm{A}$.

607 Paired-pulse facilitation was expressed as the mean ratio of second and first fEPSP 608 amplitude as a percentage with an interstimulus interval of 25, 50, 100, 150 and $200 \mathrm{~ms}$.

609 In some experiments, recombinant BDNFpro (10 ng/ml; Alomone Labs, Cat\#B-245), 610 BDNFpro ${ }^{\text {Val/Met }}(10 \mathrm{ng} / \mathrm{ml}$; Alomone Labs, Cat\#B-445), mBDNF (10 ng/ml; Laboratory 611 of Antonino Cattaneo, SNS, Pisa, Italy), proBDNF ${ }^{\mathrm{CR}}(20 \mathrm{ng} / \mathrm{ml}$; Laboratory of Antonino 612 Cattaneo, SNS, Pisa, Italy) were perfused into a recording chamber.

\section{Immunohistochemistry}

615 Brain slices were fixed in 4\% PFA for $1 \mathrm{~h}$ after recording. Slices were treated with $1 \%$ 616 Triton X-100 for 20 min, blocked with 3\% BSA in PBS for $1 \mathrm{~h}$ and incubated overnight 617 free-floating with primary antibodies diluted in blocking buffer. Slices were washed in 618 PBS and incubated for $2 \mathrm{~h}$ at room temperature with secondary antibodies diluted in 619 blocking buffer. Slices were eventually counterstained with DAPI (Sigma Aldrich, 620 Cat\#D9542) and mounted with Aqua Poly/mount (Polysciences, Inc., Cat \#18606).

\section{Antibodies}


623 The following antibodies were used: rabbit $\alpha$-GFP (Thermo Fisher Scientific Cat\#A624 6455; RRID:AB\#2536208; IHC 1:1000 ICC 1:1000), chicken $\alpha$-GFP (Thermo Fisher 625 Scientific Cat\#A10262; RRID:AB\#2534023, IHC 1:1000), chicken $\alpha$-BDNF (Promega 626 Cat\#G1641; RRID:AB\#430850, IHC 1:300), rabbit $\alpha$-BDNF (Alomone Labs Cat\# ANT627 010; RRID:AB_2039756, EM 1:20), chicken $\alpha$-proBDNF (Millipore Cat\#AB9042; 628 RID:AB\#2274709, IHC 1:300), rabbit $\alpha$-proBDNF (Alomone Labs Cat\#ANT-006; 629 RRID:AB_2039758, EM 1:20), rabbit $\alpha$-BDNFpro (Laboratory of Bai Lu, Govern 630 Institute for Brain Research, Tsinghua University, Beijing, IHC 1:300, EM 1:20, WB 631 1:500), mouse $\alpha-N e u N$ (Abcam Cat\#ab77315; RRID:AB\#1566475, IHC 1:1000), guinea 632 pig $\alpha-N e u N$ (Millipore Cat\#ABN90; RRID:AB\#11205592, IHC 1:1000), rabbit $\alpha$-pTrkB 633 (Tyr 816) (Laboratory of Moses Chao, Skirball Intitute of Biomolecular Medicine, New 634 York, USA, IHC 1,25 mg/ml), goat $\alpha$-TrkB (Santa Cruz Biotechnology Cat\#sc-12-G; 635 RRID:AB\#632558, IHC 1:300), mouse $\alpha$-PSD95 (Merck-Millipore Cat\#MAB1596; 636 RRID:AB_2092365, IHC 1:500), sheep $\alpha$-SorCS2 (R\&D system Cat\#AF4238; 637 RRID:AB_10645642), rabbit $\alpha$-SorCS2 (MyBioSource Cat\# MBS5302436), rabbit $\alpha$ 638 p75 ${ }^{\text {NTR }}$ (Promega Cat\#G3231; RRID:AB_430853, IHC 1:1000), chicken $\alpha$-GFAP 639 (Abcam Cat\#ab134436; RRID:AB_2818977, IHC 1:1000), mouse $\alpha$-GFAP (Abcam 640 Cat\#ab10062; RRID:AB_296804, IHC 1:1000), mouse $\alpha$-synaptobrevin2lVAMP2 641 (Synaptic System Cat\#104 211; RRID:AB_2619758, IHC 1:300), rabbit $\alpha$-beta 642 galactosidase (Proteintech Cat\#15518-1-AP; RRID:AB_2263448, IHC 1:500), rabbit $\alpha$ 643 RFP (Rockland Antibodies Cat\#600-401-379; RRID:AB_2209751 IHC 1:1000), mouse $\alpha$ 644 DsRed (Santa Cruz Biotechnology Cat\# sc-390909; RRID:AB_2801575 IHC 1:1000).

\section{Western blot experiments}

647 Recombinant $\mathrm{mBDNF}$, proBDNF${ }^{\mathrm{CR}}$ and BDNFpro were suspended in protein sample 648 buffer, boiled for $7 \mathrm{~min}$ at $95^{\circ} \mathrm{C}$, resolved by $12 \%$ SDS-PAGE and transferred to 649 nitrocellulose membranes. Membranes were incubated $(1 \mathrm{~h})$ at room temperature in 650 blocking solution (5\% BSA in TBS-Tween 0.1\%) and incubated overnight at $4^{\circ} \mathrm{C}$ with 651 primary antibodies. Membranes were washed 3 times in TBS-Tween and incubated for 
$1.5 \mathrm{~h}$ at room temperature with horseradish peroxidase (HRP)-conjugated secondary

653 antibodies. ECL was used for detection.

654

655 Primary cell cultures

656 Cortices were isolated from E17.5 mouse embryos and incubated for $20 \mathrm{~min}$ at $37^{\circ} \mathrm{C}$ in 657 Trypsin/EDTA $0.25 \%$ (Thermo Fisher Scientific, Cat\#25200056). Cortical cells were 658 dissociated with a plastic pipette, collected by centrifugation (800 RPM, 5 min) and 659 resuspended in Dulbecco's modified Eagle's medium (DMEM; GIBCO Cat\#10938025) 660 supplemented with $10 \%$ of fetal bovine serum and Penicillin/Streptomycin (GIBCO; 661 Cat\#15070063). Cells were transfected using Amaxa Nucleofector system following 662 manufacturer instructions and plated on glass coverslips precoated with poly-L-lysine 663 (0.1 mg/ml; Sigma Aldrich, Cat\#P6282). Three hours after plating, the medium was 664 changed to Neurobasal (GIBCO, Cat\#21103049) supplemented with B27-supplement 665 (GIBCO, Cat\#17504044) and Penicillin/Streptomycin/Glutamine (GIBCO, 666 Cat\#1038016).

667

668 Proximity ligation assay (PLA)

669 PLA was performed on fixed mouse primary cortical neurons non-permeabilized and on 670 fixed perirhinal sections permeabilized with Triton X $1 \%$ for 20 minutes. PLA was 671 performed following manufacturer instruction (Duolink PLA Sigma Aldrich). Briefly 672 samples were blocked with 3\% BSA in PBS for 1 hour and incubated with primary 673 antibodies at $4^{\circ} \mathrm{C}$ overnight. Samples were washed with PBS and incubated with PLA 674 probes MINUS (Duolink PLA Probe Anti-Goat MINUS antibody, Sigma Aldrich Cat\# 675 DUO92006) and PLUS (Duolink PLA Probe Anti-Rabbit PLUS antibody, Sigma Aldrich 676 Cat\# DUO92002) for $1 \mathrm{~h}$ at $37^{\circ} \mathrm{C}$. The PLA probes were diluted (1:5) in antibody diluent. 677 Samples were washed in 1X buffer A 2X5 min under gentle agitation and incubated with 678 ligation- ligase solution for $30 \mathrm{~min}$ at $37^{\circ} \mathrm{C}$. Samples were washed in $1 \mathrm{X}$ wash buffer for $6792 \times 2$ min under gentle agitation and incubated with amplification polymerase solution for $68090 \mathrm{~min}$ at $37^{\circ} \mathrm{C}$. In some experiments, we performed immunodetection over PLA 681 incubating PLA samples with secondary antibodies coupled with the appropriate 
$6832 \mathrm{X} 10 \mathrm{~min}$ and with $0.01 \mathrm{X}$ wash buffer B for $1 \mathrm{~min}$. Samples were mounted with 684 Prolong Gold antifade reagent with DAPI.

\section{Confocal microscopy and quantitative image analysis}

687 Confocal imaging was performed using a laser-scanning motorized confocal system 688 (Nikon A1) equipped with an Eclipse Ti-E inverted microscope and four laser lines (405, 689488,561 and $638 \mathrm{~nm}$ ). Z-series images were taken with an inter-stack interval of $0.5 \mu \mathrm{m}$ 690 using $60 \mathrm{X}$ oil objective. For each type of quantification, laser intensities and camera 691 settings were maintained identically within the same experiment to allow the comparison 692 of different experimental groups and treatments. Image processing and 3D-rendering 693 were performed using the software NIS Element (Nikon). Colocalizations 694 (BDNFpro/GFP; Vamp2-GFP; BDNFpro/Vamp2; TrkB/pTrkB; PLA ${ }^{\text {TrkB/SorCS2 }} / \mathrm{NeuN}$; 695 PLA $^{\text {TrkB/SorCS2} / P S D 95) ~ w e r e ~ q u a n t i f i e d ~ c a l c u l a t i n g ~ M a n d e r ' s ~ o v e r l a p ~ o r ~ P e a r s o n ' s ~}$ 696 correlation using the software NIS Element (Nikon). Colocalization data for the 697 comparison of stimulated slices (TBS) vs. non-stimulated slices (baseline) are expressed 698 following normalization of the Mander's overlap of each treatment to the average of 699 Mander's overlap of the baseline.

\section{D-SIM}

702

3D-SIM was performed using X-Light V2 confocal spinning disk system completed with a Video Confocal super-resolution module (CrestOptics, Italy) with a lateral resolution of $04115 \mathrm{~nm}$ and an axial resolution of $\sim 250 \mathrm{~nm}$. The system was equipped with a $60 \mathrm{x} / 1.40$ 5 NA PlanApo Lambda oil immersion objective (Nikon, Japan), Zyla sCMOS camera 06 (Andor) and Spectra X Lumencor LEDs Light Source with bandpass excitation filters of 460-490 and 535-600 nm (Chroma Technology, US). Image stacks were acquired with a 08 format of $2048 \times 2048$ pixels and a z-distance of $150 \mathrm{~nm}$ and with 36 raw SIM images per 09 plane (multiple acquisition mode $x-y$ grid scan). The SIM raw data with 16-bit depth 711 image rendering and colocalization analysis, images were processed using NIS-Elements 
712 software. Quantification of BDNFpro/GFP puncta was performed manually counting the 713 number of immunoreactive puncta in finger-like extension and lamellar sheaths compared 714 to the total number of puncta found in astrocytes (for these experiments $n=6$ cells, 3 715 slices, 3 mice for each condition).

\section{Electron microscopy}

718 Sections $(300 \mu \mathrm{m})$ from electrophysiological recordings were fixed in a solution of $4 \%$ 719 PFA and $0.1 \%$ glutaraldehyde in $0.1 \mathrm{M}$ phosphate buffer (PB) for 4 hours and then 720 washed overnight in $50 \mathrm{mM}$ TBS, $\mathrm{pH}$ 7.4. Slices were immersed for $2 \mathrm{~h}$ in a 721 cryoprotecting solution (25\% sucrose and $10 \%$ glycerol in $0.05 \mathrm{M} \mathrm{PB}$ ) before being snap 722 frozen in liquid nitrogen-cooled isopentane. After washing in 0.1 M PB, sections were 723 incubated for $1 \mathrm{~h}$ in $20 \%$ normal goat serum in TBS. Slices were then incubated for three 724 nights at $4^{\circ} \mathrm{C}$ in a $3 \% \mathrm{BSA} / \mathrm{TBS}$ solution containing primary $\alpha \mathrm{BDNF}$ pro or $\alpha \mathrm{mBDNF}$ or 725 aproBDNF. Sections were incubated overnight at $4^{\circ} \mathrm{C}$ in $3 \%$ BSA/TBS solution 726 containing $2 \mathrm{~nm}$-gold conjugated goat arabbit (BBI International). Sections were rinsed in 727 TBS and then fixed for $30 \mathrm{~min}$ in a 2,5\% glutaraldehyde solution and post-fixed with $1 \%$ 728 osmium tetroxide. After dehydration in an ethanol series followed by propylene oxide, 729 samples were embedded in Epon812 epoxy resin and observed with a JEOLJEM-1011 730 transmission electron microscope, operated at $100 \mathrm{kV}$, after cutting ultrathin sections (60 $731 \mathrm{~nm}$ thick). Negative controls were performed in the absence of primary antibody.

732 Astrocytes were identified using 3 structural criteria: (i) localization between synapses: 733 the space between synapses is occupied by astrocytic processes. Astrocytes are indeed 734 cellular structures just filling all the space between synapses, dendrites, and axons; (ii) 735 irregular, stellate shape: the processes of astrocytes surrounding synaptic contacts often 736 adopt quite convoluted forms. In comparison to their flatter neuronal counterparts, 737 perisynaptic astrocytes show an irregular profile; (iii) relatively clear cytoplasm: the 738 cytoplasm of an astrocyte differs from that of neighboring objects in the neuropil. An 739 astrocyte cytoplasm is less electron-dense than the one of neurons. Quantification of gold 740 grains was performed manually counting the number of gold grains in a membrane 
741 delimited area of $230 \mathrm{~nm}$ radius surrounding synaptic contacts compared to the total 742 number of gold grains found in astrocytes (for these experiments $n=5$ slices; 3 mice).

\section{Object recognition test (ORT)}

745 ORT was performed in a Y-apparatus with high, homogenous white walls, $30 \mathrm{~cm}$ high:

746 one arm was used as start arm and had a sliding door to control access to the arena; the 747 other two arms were used to display the objects. The start arm is $26 \mathrm{~cm}$ in length with the 748 sliding door placed at $13 \mathrm{~cm}$, and the lateral arms are $18 \mathrm{~cm}$ long. All arms are $10 \mathrm{~cm}$ 749 wide. On the first day (habituation day) mice explored the arena for $20 \mathrm{~min}$. The 750 following day, the learning session (sample phase) was performed: for five minutes the 751 animals were free to explore the arena, which contained two identical objects, one for 752 each arm, placed at the end of the arm. At $10 \mathrm{~min}$ or $24 \mathrm{~h}$ after the sample phase, the test 753 phase was run; the animals were free to explore the arena containing two objects, the 754 familiar object and a novel one. Arena and objects were cleaned up between trials to stop 755 the build-up of olfactory cues. The discrimination index was calculated as follows: 756 (TNew - TOld)/(TNew + TOld), with TNew and TOld being the time spent exploring the 757 new and the familiar objects, respectively. A video camera was mounted above the 758 apparatus to record trials with the EthoVision software (Noldus). The exploration time 759 was taken as the time during which mice approached the objects with muzzle and paws.

\section{Statistics and reproducibility}

762 All data were run for a normality test before a statistical comparison test. Data normally 763 distributed were summarized by mean \pm SEM. Comparison between two different groups 764 was assessed by an unpaired t-test. For ORT discrimination index, two-way ANOVA 765 followed by a Holm-Sidak method test was used. The level of significance used was $\mathrm{p}<$ 7660.05 .

\section{Data availability}

769 The authors declare that all data supporting the findings of this study are available within 770 the paper and its supplementary information files. 


\section{References}

1. F. Crick, Neurobiology: Memory and molecular turnover. Nature 312, 101-101 (1984).

2. J. E. Lisman, M. A. Goldring, Feasibility of long-term storage of graded information by the $\mathrm{Ca} 2+/$ calmodulin-dependent protein kinase molecules of the postsynaptic density. Proc. Natl. Acad. Sci. 85, 5320-5324 (1988).

3. K. Kim, T. Saneyoshi, T. Hosokawa, K. Okamoto, Y. Hayashi, Interplay of enzymatic and structural functions of CaMKII in long-term potentiation. $J$. Neurochem. 139, 959-972 (2016).

4. S. Miller, Regulation of brain Type II Ca2+/calmodulin-dependent protein kinase by autophosphorylation: A Ca2+-triggered molecular switch. Cell 44, 861-870 (1986).

5. K. Si, E. R. Kandel, The Role of Functional Prion-Like Proteins in the Persistence of Memory. Cold Spring Harb. Perspect. Biol. 8, a021774 (2016).

6. R. A. Nicoll, A Brief History of Long-Term Potentiation. Neuron 93, 281-290 (2017).

7. N. J. Allen, Astrocyte regulation of synaptic behavior. Annu. Rev. Cell Dev. Biol. 30, 439-63 (2014).

8. A. Araque, et al., Gliotransmitters travel in time and space. Neuron 81, 728-739 (2014).

9. R. D. Fields, et al., Glial Biology in Learning and Cognition. Neurosci. 20, 426431 (2014).

10. V. Lessmann, T. Brigadski, Mechanisms, locations, and kinetics of synaptic BDNF secretion: an update. Neurosci. Res. 65, 11-22 (2009).

11. H. Park, M. Poo, Neurotrophin regulation of neural circuit development and function. Nat. Rev. Neurosci. 14, 7-23 (2013).

12. B. Lu, P. T. Pang, N. H. Woo, The yin and yang of neurotrophin action. Nat. Rev. Neurosci.6, 603-14 (2005).

13. M. Bergami, et al., Uptake and recycling of pro-BDNF for transmitter-induced secretion by cortical astrocytes. J. Cell Biol. 183, 213-221 (2008).

14. S. Manju, B. Vignoli, M. Canossa, R. Blum, Neurobiology of local and intercellular BDNF signaling. Pflugers Arch. Eur. J. Physiol. (2017).

15. B. Vignoli, et al., Peri-Synaptic Glia Recycles Brain-Derived Neurotrophic Factor for LTP Stabilization and Memory Retention. Neuron 92 (2016).

16. D. Lanjakornsiripan, et al., Layer-specific morphological and molecular differences in neocortical astrocytes and their dependence on neuronal layers. Nat. Commun. 9, 1623 (2018).

17. B. Vignoli, M. Canossa, Glioactive ATP controls BDNF recycling in cortical astrocytes. Commun. Integr. Biol. 10 (2017).

18. J. Boutilier, et al., Proneurotrophins require endocytosis and intracellular 
proteolysis to induce TrkA activation. J. Biol. Chem. 283, 12709-16 (2008).

19. H. Rösch, R. Schweigreiter, T. Bonhoeffer, Y.-A. Barde, M. Korte, The neurotrophin receptor p75NTR modulates long-term depression and regulates the expression of AMPA receptor subunits in the hippocampus. Proc. Natl. Acad. Sci. U. S. A. 102, 7362-7 (2005).

20. N. H. Woo, et al., Activation of p75NTR by proBDNF facilitates hippocampal long-term depression. Nat. Neurosci. 8, 1069-77 (2005).

21. P. I. Ortinski, et al., Selective induction of astrocytic gliosis generates deficits in neuronal inhibition. Nat. Neurosci. 13, 584-591 (2010).

22. L. Zhuo, et al., Live Astrocytes Visualized by Green Fluorescent Protein in Transgenic Mice. Dev. Biol. 187, 36-42 (1997).

23. G. Aicardi, et al., Induction of long-term potentiation and depression is reflected by corresponding changes in secretion of endogenous brain-derived neurotrophic factor. Proc. Natl. Acad. Sci. U. S. A. 101, 15788-92 (2004).

24. P. T. Pang, G. Nagappan, W. Guo, B. Lu, Extracellular and intracellular cleavages of proBDNF required at two distinct stages of late-phase LTP. npj Sci. Learn. 1, 16003 (2016).

25. J. S. Aaron, A. B. Taylor, T.-L. Chew, Image co-localization - co-occurrence versus correlation. J. Cell Sci. 131, jcs211847 (2018).

26. V. Lessmann, K. Gottmann, M. Malcangio, Neurotrophin secretion: current facts and future prospects. Prog. Neurobiol. 69, 341-374 (2003).

27. E. Bogenmann, et al., Generation of mice with a conditional allele for the p75(NTR) neurotrophin receptor gene. Genesis 49, 862-869 (2011).

28. T. Mori, et al., Inducible gene deletion in astroglia and radial glia--a valuable tool for functional and lineage analysis. Glia 54, 21-34 (2006).

29. P. Soriano, Generalized lacZ expression with the ROSA26 Cre reporter strain. Nat. Genet. 21, 70-71 (1999).

30. E. Zuccaro, et al., Polarized expression of p75(NTR) specifies axons during development and adult neurogenesis. Cell Rep. 7, 138-52 (2014).

31. J. Grosche, et al., Microdomains for neuron-glia interaction: parallel fiber signaling to Bergmann glial cells. Nat. Neurosci. 2, 139-143 (1999).

32. B. Fernandez, I. Suarez, G. Gonzalez, Topographical distribution of the astrocytic lamellae in the hypothalamus. Anat. Anz. 156, 31-7 (1984).

33. B. S. Khakh, M. V Sofroniew, Diversity of astrocyte functions and phenotypes in neural circuits. Nat. Neurosci. 18, 942-952 (2015).

34. I. Patrushev, N. Gavrilov, V. Turlapov, A. Semyanov, Subcellular location of astrocytic calcium stores favors extrasynaptic neuron-astrocyte communication. Cell Calcium 54, 343-349 (2013).

35. N. Gavrilov, et al., Astrocytic Coverage of Dendritic Spines, Dendritic Shafts, and Axonal Boutons in Hippocampal Neuropil. Front. Cell. Neurosci. 12 (2018).

36. V. Montana, E. B. Malarkey, C. Verderio, M. Matteoli, V. Parpura, Vesicular 
transmitter release from astrocytes. Glia 54, 700-715 (2006).

854 37. S. Dieni, et al., BDNF and its pro-peptide are stored in presynaptic dense core vesicles in brain neurons. J. Cell Biol. 196, 775-88 (2012).

38. M. F. Egan, et al., The BDNF val66met Polymorphism Affects ActivityDependent Secretion of BDNF and Human Memory and Hippocampal Function. Cell 112, 257-269 (2003).

39. S.-J. Tsai, Critical Issues in BDNF Val66Met Genetic Studies of Neuropsychiatric Disorders. Front. Mol. Neurosci. 11 (2018).

40. Z.-Y. Chen, Variant Brain-Derived Neurotrophic Factor (BDNF) (Met66) Alters the Intracellular Trafficking and Activity-Dependent Secretion of Wild-Type BDNF in Neurosecretory Cells and Cortical Neurons. J. Neurosci. 24, 4401-4411 (2004).

41. A. Anastasia, et al., Val66Met polymorphism of BDNF alters prodomain structure to induce neuronal growth cone retraction. Nat. Commun. 4, 2490 (2013).

42. S. Glerup, et al., SorCS2 is required for BDNF-dependent plasticity in the hippocampus. Mol. Psychiatry 21, 1740-1751 (2016).

43. M. W. Brown, J. P. Aggleton, Recognition memory: what are the roles of the perirhinal cortex and hippocampus? Nat. Rev. Neurosci. 2, 51-61 (2001).

44. E. A. Murray, T. J. Bussey, L. M. Saksida, Visual perception and memory: a new view of medial temporal lobe function in primates and rodents. Annu. Rev. Neurosci. 30, 99-122 (2007).

45. T. J. Kettenmann H, Faissner A, "Comprehensive Human Physiology" in R. Greger, U. Windhorst, Eds. (Springer Berlin Heidelberg, 1996).

46. A. Araque, R. P. Sanzgiri, V. Parpura, P. G. Haydon, Astrocyte-induced modulation of synaptic transmission. Can. J. Physiol. Pharmacol. 77, 699-706 (1999).

47. M. A. Di Castro, et al., Local Ca2+ detection and modulation of synaptic release by astrocytes. Nat. Neurosci. 14, 1276-1284 (2011).

48. J. Grosche, H. Kettenmann, A. Reichenbach, Bergmann glial cells form distinct morphological structures to interact with cerebellar neurons. J. Neurosci. Res. 68, 138-149 (2002).

49. I. Polykretis, V. Ivanov, K. P. Michmizos, "The Astrocytic Microdomain as a Generative Mechanism for Local Plasticity" in In: Wang S. et al., Eds. (Brain Informatics. BI, Lecture Notes in Computer Science, vol 11309. Springer, Cham., 2018).

50. B. Scholl, D. E. Wilson, D. Fitzpatrick, Local Order within Global Disorder: Synaptic Architecture of Visual Space. Neuron 96, 1127-1138.e4 (2017).

51. A. Govindarajan, R. J. Kelleher, S. Tonegawa, A clustered plasticity model of long-term memory engrams. Nat. Rev. Neurosci. 7, 575-83 (2006).

52. P. Poirazi, B. W. Mel, Impact of Active Dendrites and Structural Plasticity on the Memory Capacity of Neural Tissue. Neuron 29, 779-796 (2001). 
53. T. Mizui, et al., BDNF pro-peptide actions facilitate hippocampal LTD and are altered by the common BDNF polymorphism Val66Met. Proc. Natl. Acad. Sci. 112, E3067-E3074 (2015).

54. M. Song, K. Martinowich, F. S. Lee, BDNF at the synapse: why location matters. Mol. Psychiatry 22, 1370-1375 (2017).

55. A. K. Fu, N. Y. Ip, Regulation of postsynaptic signaling in structural synaptic plasticity. Curr. Opin. Neurobiol. 45, 148-155 (2017).

56. L. Minichiello, et al., Mechanism of TrkB-mediated hippocampal long-term potentiation. Neuron 36, 121-37 (2002).

57. K.-O. Lai, et al., TrkB phosphorylation by Cdk5 is required for activity-dependent structural plasticity and spatial memory. Nat. Neurosci. 12 (2012).

58. U. Frey, R. G. Morris, Synaptic tagging and long-term potentiation. Nature 385, 533-6 (1997).

59. R. G. M. Morris, Elements of a neurobiological theory of hippocampal function: the role of synaptic plasticity, synaptic tagging and schemas. Eur. J. Neurosci. 23, 2829-2846 (2006).

60. Y. Lu, et al., TrkB as a Potential Synaptic and Behavioral Tag. J. Neurosci. 31, 11762-11771 (2011).

61. D. O. Hebb, The organization of behavior; a neuropsychological theory (Wiley, 1949).

62. E. Blanco-Suárez, A. L. M. Caldwell, N. J. Allen, Role of astrocyte-synapse interactions in CNS disorders. J. Physiol. 595, 1903-1916 (2017).

63. M. Poo, et al., What is memory? The present state of the engram. BMC Biol. 14, 40 (2016).

64. A. Adamsky, I. Goshen, Astrocytes in Memory Function: Pioneering Findings and Future Directions. Neuroscience 370, 14-26 (2018).

65. P. J. Gebicke-Haerter, Engram formation in psychiatric disorders. Front. Neurosci. 8 (2014).

66. L. E. Clarke, B. A. Barres, Emerging roles of astrocytes in neural circuit development. Nat. Rev. Neurosci. 14, 311-321 (2013). 


\section{Acknowledgments}

927 We thank Massimo Gamberini and Silvia Biggi for technical assistance and Ranzi 928 Barbara for lab management; Moses Chao for providing pTrkB antibody; Brian A. 929 Pierchala for providing $\mathrm{p} 75^{\text {lox/lox }}$ mice; Antonino Cattaneo for providing recombinant 930 BDNF and proBDNF ${ }^{\mathrm{CR}}$; Magdalena Götz for providing GLAST-CreER ${ }^{\mathrm{T} 2}$ mice; Annalisa 931 Buffo for providing GFAP-GFP mice; IN-BDNF consortium for scientific support.

932 This project received founding from Progetti di Ricerca di Rilevanza Nazionale (PRIN), 933 Bando 2017 Project number 2017HPTFFCPRIN to M.C.; European Research Council 934 (ERC) under the European Union's Horizon 2020 research and innovation program 935 (grant agreement $\mathrm{N}^{\circ}$ 788793-BACKUP) to M.C. and B.V.; Deutsche 936 Forschungsgemeinschaft BL567/3- 2 and project number 44541416-TRR58-A10 to 937 R.B.; Graduate School of Life Sciences (GSLS) Würzburg fellowship to M.S.; 938 Fondazione Umberto Veronesi post-doctoral grant 2018 to B.V.

\section{Author contributions}

941 Conceptualization: B.V. and M.C.; Investigation; B.V., S.S., M.S., and G.S.; Writing942 original draft: B.V. and M.C.; Writing-review and editing; R.B. and B.L.; Supervision: 943 R.B., S.S. and N.B.; Funding acquisition: M.C., B.V. and R.B.; Resources: B.L., R.B and 944 S.S.

945

946 Competing interest

947 The authors declare no competing interests. 


\section{Figures}

a

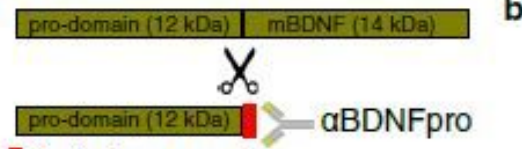

furin cleavage site

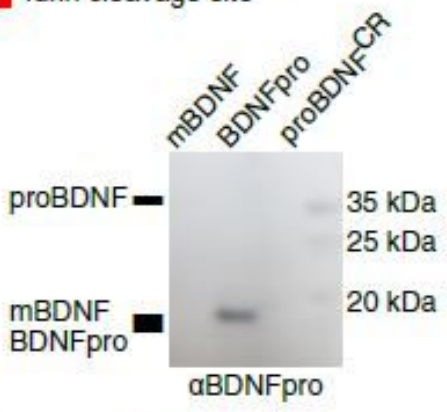

c

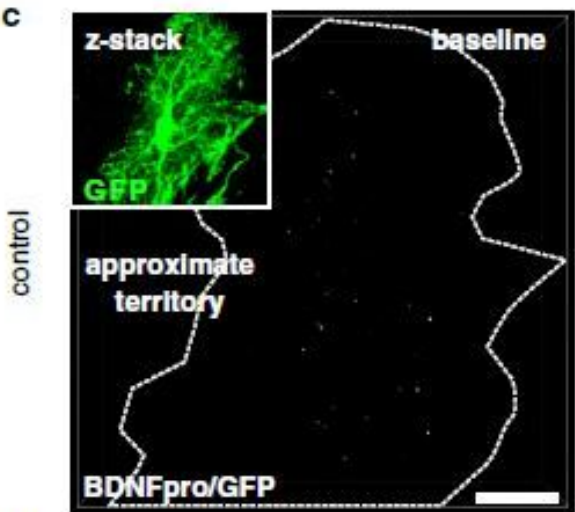

d

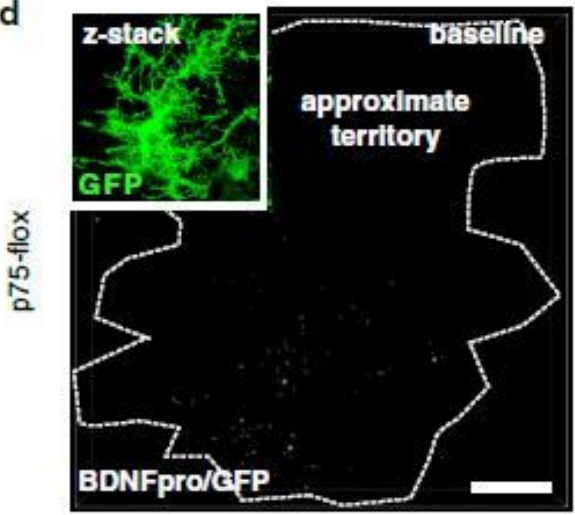

b

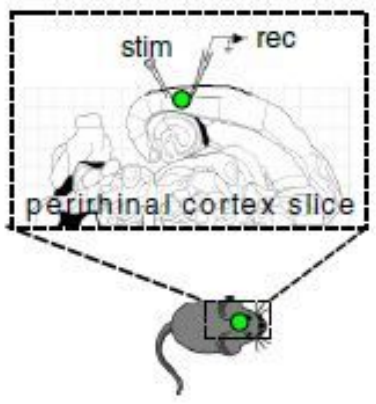

O injection site
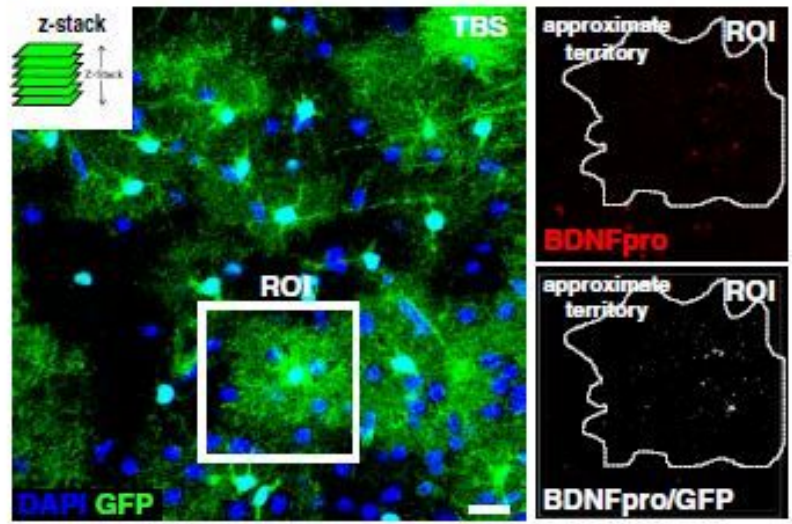

BDNFpro/GFP
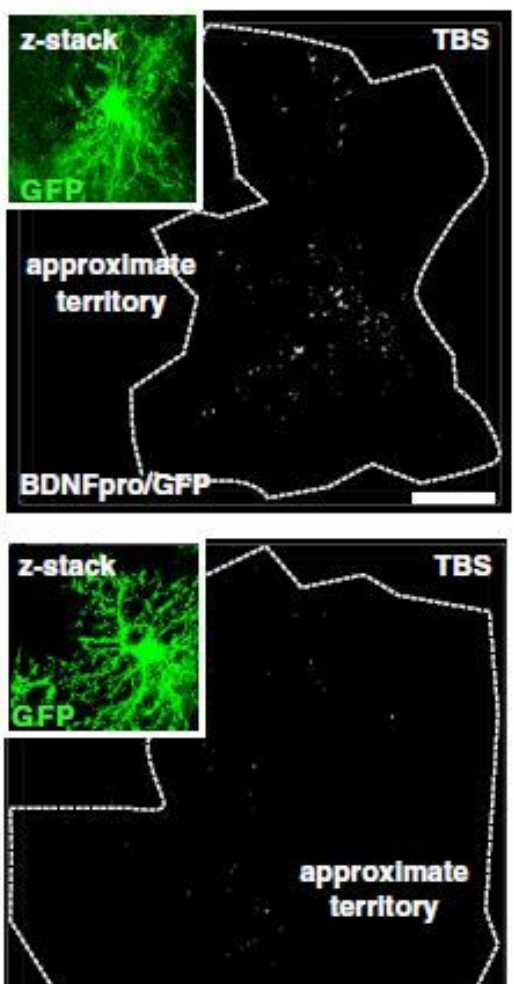

BDNPprodere

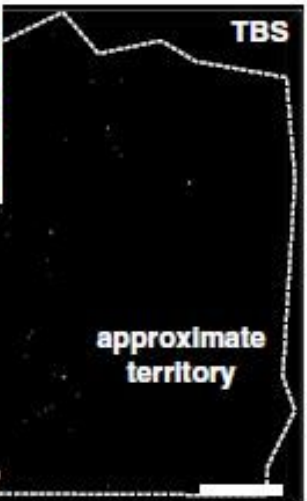

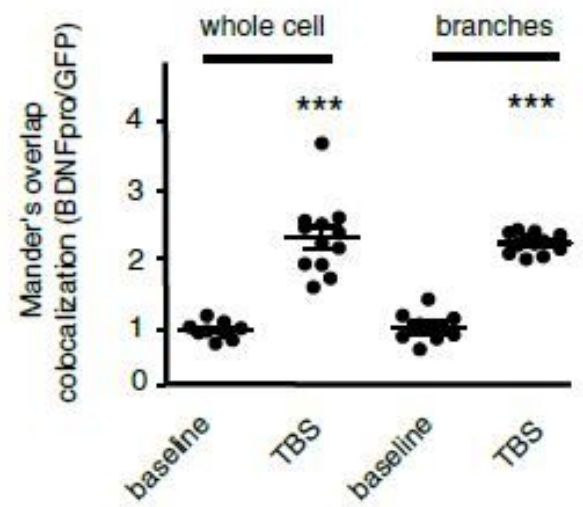

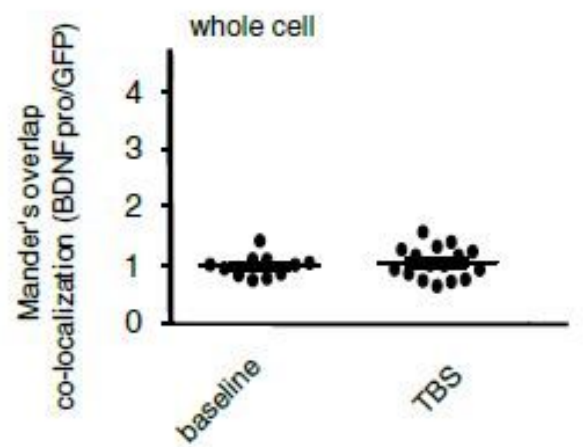

\section{Figure 1}

proBDNF processing in cortical astrocytes (a) Schematic representation of proBDNF precursor and cleaved BDNFpro domain (upper). aBDNFpro antibody recognizes the furin cleavage site of the prodomain. Western blotting probing recombinant mBDNF, BDNFpro and proBDNFCR with aBDNFpro antibody (lower). (b) Cortical slices from control mice injected with AAV-GFAP-GFP virus were recorded and fixed $10 \mathrm{~min}$ after TBS for immunostaining (left). z-stack reconstruction shows astrocytes labeled by GFP (middle). Three times magnification of a single stack from a region of interest (ROI) (right) show 
BDNFpro immunoreactivity (upper) and BDNFpro/GFP colocalization signal (lower) of one GFP-astrocyte delimited by an approximate territory (white dashed). Scale bar: $10 \mu \mathrm{m}$. (c) z-stack reconstruction of BDNFpro/GFP colocalization signals in astrocytes from baseline- and TBS-slices from control mice. The insets show GFP signal. BDNFpro/GFP colocalization was quantified in the whole cell and branches using Mander's overlap. Data are normalized to baseline and presented as mean \pm SEM. $* * * p<0.001$ (Unpaired t-test); ( $n=9$ cells, 4 slices, 3 mice for baseline; $n=12$ cells, 4 slices, 3 mice for TBS). Scale bars: $10 \mu \mathrm{m}$. (d) z-stack reconstruction of BDNFpro/GFP colocalization signals in astrocytes from basaland TBS-slices from tamoxifen-treated p75-flox mice. Insets show GFP signal. BDNFpro/GFP colocalization was quantified using Mander's overlap. Data are normalized to baseline and presented as mean \pm SEM. ( $n=11$ cells, 5 slices, 4 mice for baseline; $n=16$ cells, 5 slices, 4 mice for TBS). Scale bar: $10 \mu \mathrm{m}$.

a Structured Illumination Microscopy

(SIM)

Super-resolution of VCS Module (CrestOptics)

Metamorph and NIS Software 36 raw SIM images per plane
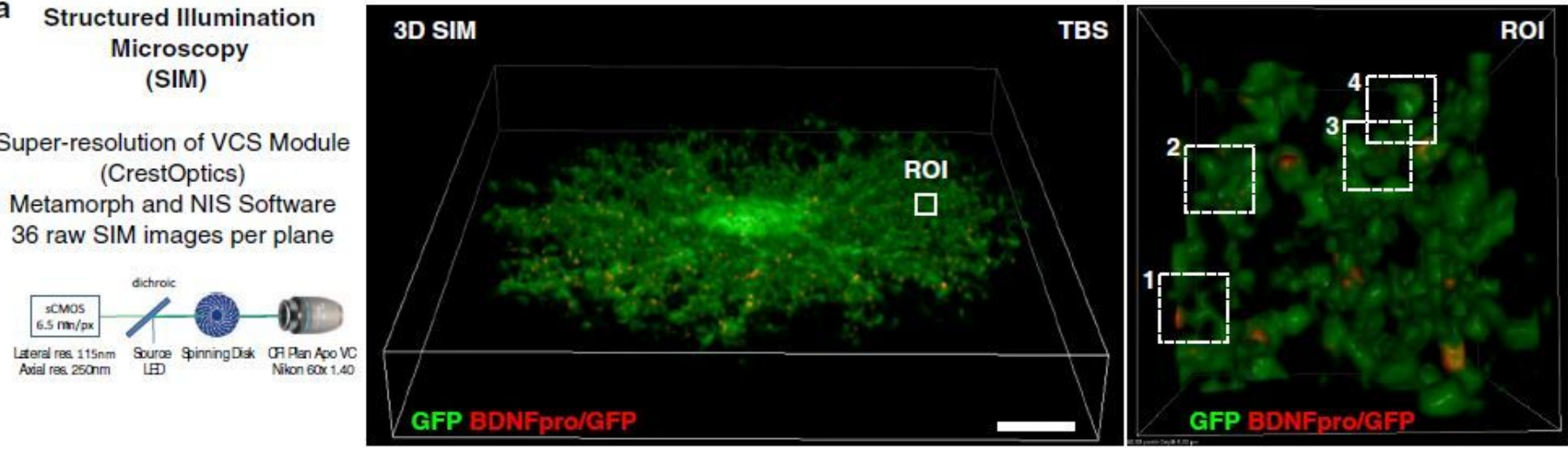

b
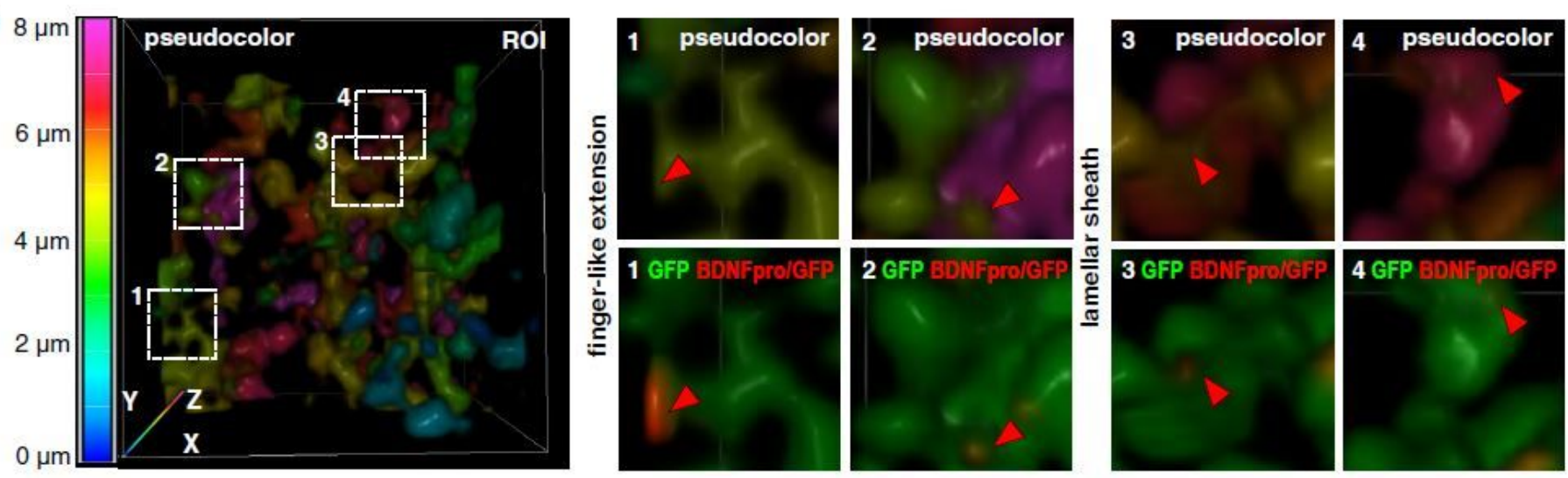

\section{Figure 2}

subcellular localization of BDNFpro (a) Graphical representation of the SIM super-resolution microscope (left). 3D-SIM image of a GFP-labeled astrocyte in a TBS-slice from control mice (middle). Twenty times magnification of a ROI shows BDNFpro/GFP colocalization signal localized in fine membrane extensions of the cell periphery (right). Scale bar: $10 \mu \mathrm{m}$. (b) 3D-SIM image of the ROI in (a); z-axe is visualized in pseudocolor to facilitate microdomains identification. Three times magnification of microdomains 
characterized by the typical fingerlike extension (dashed squares 1 and 2) and flat lamellar sheath (dashed squares 3 and 4) are shown. BDNFpro/GFP colocalization is indicated (red arrowheads).

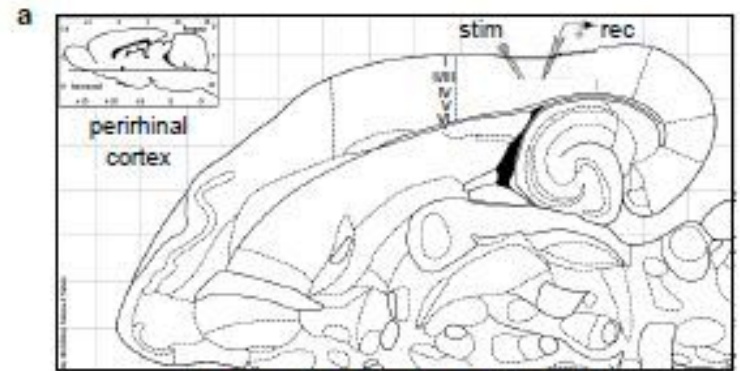

Interaural $2.66 \mathrm{~mm}$; Bregma - $7.34 \mathrm{~mm}$

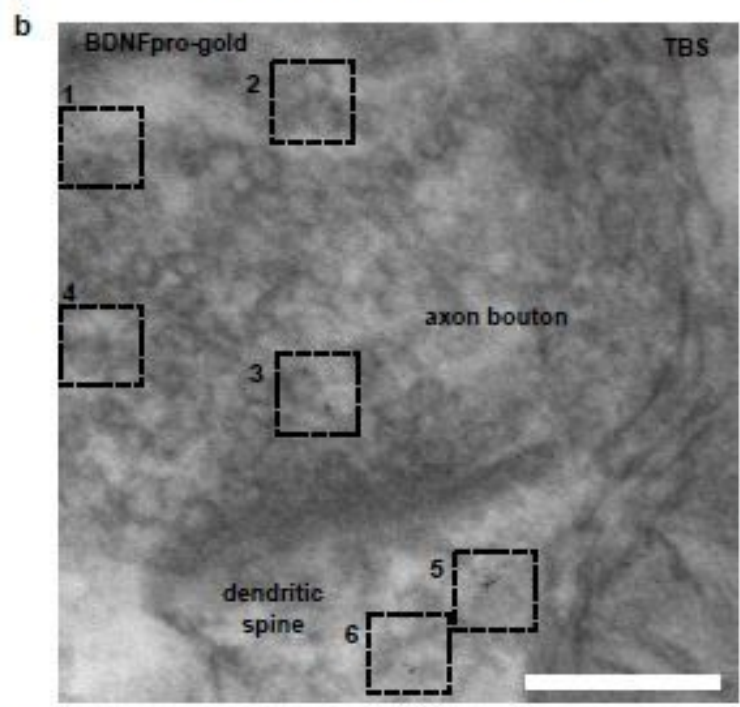

c

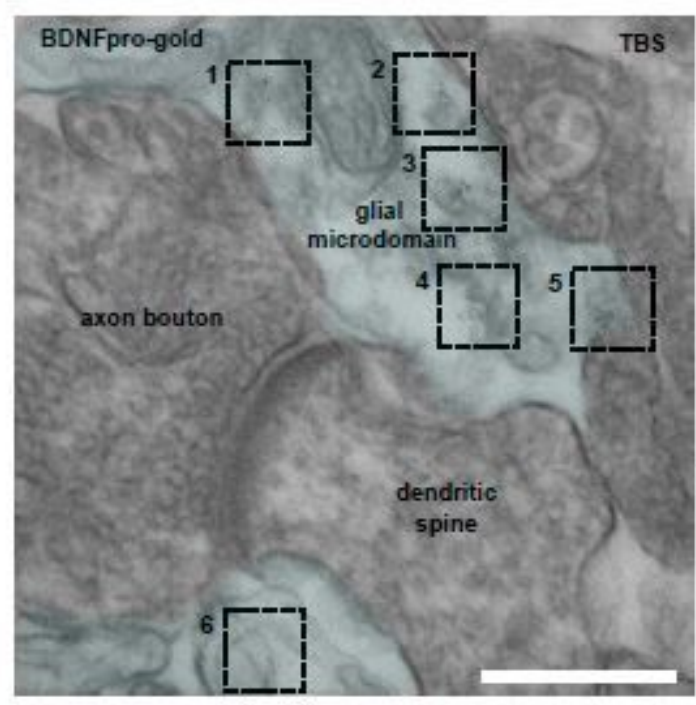

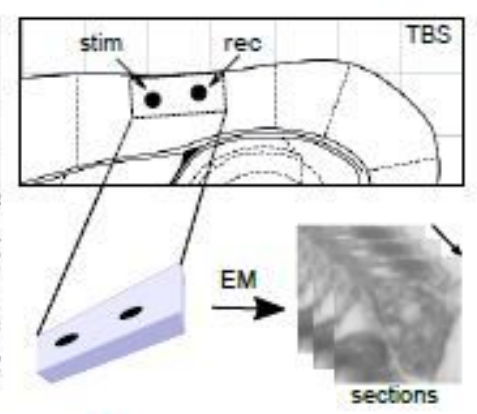
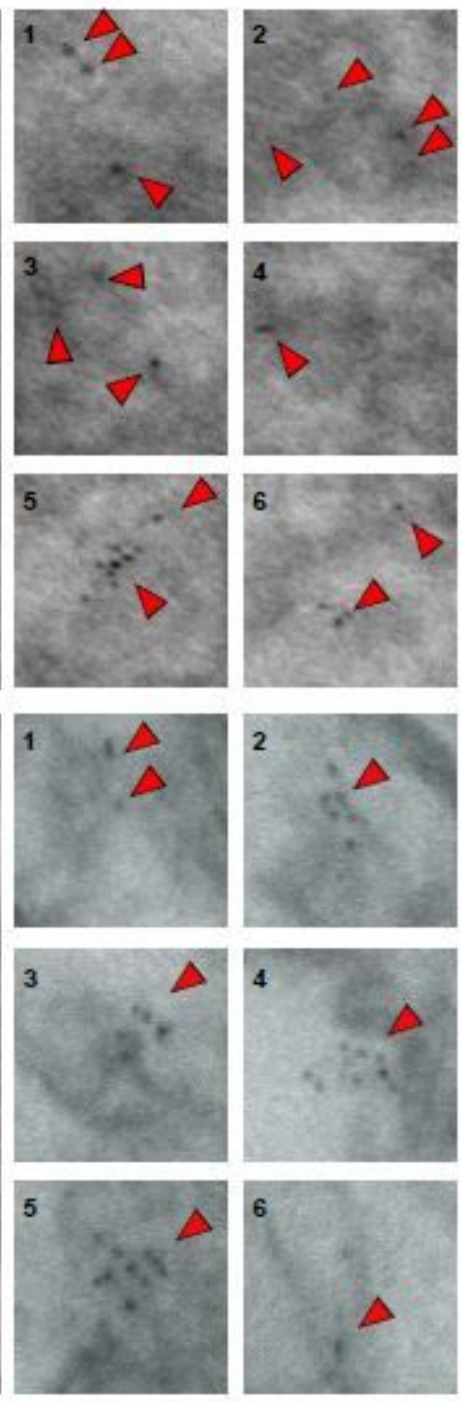

\section{Figure 3}

localization of BDNFpro in astrocytic microdomains (a) Experimental design linking field-potential with electron microscopy (EM) in layer II/III perirhinal cortex. TBS (10 min)-slices were dissected for EM processing. (b) Representative EM-image depicts BDNFpro-gold particles at axon bouton (dashed squares 1 to 4 ) and dendritic spine (dashed squares 5 and 6) (left). Three times magnification indicates 
representative areas (dashed square 1 to 6 ) in which gold particles (red arrowheads) localization is shown (right). Scale bar: $100 \mathrm{~nm}$. (c) Representative EM-image depicts BDNFpro-gold particles (dashed square 1 to 6 ) at astrocytic microdomains (light blue) (left). Three times magnification indicates representative areas (dashed square 1 to 6 ) in which gold particles (red arrowheads) localization is shown (right). Scale bar: $250 \mathrm{~nm}$.
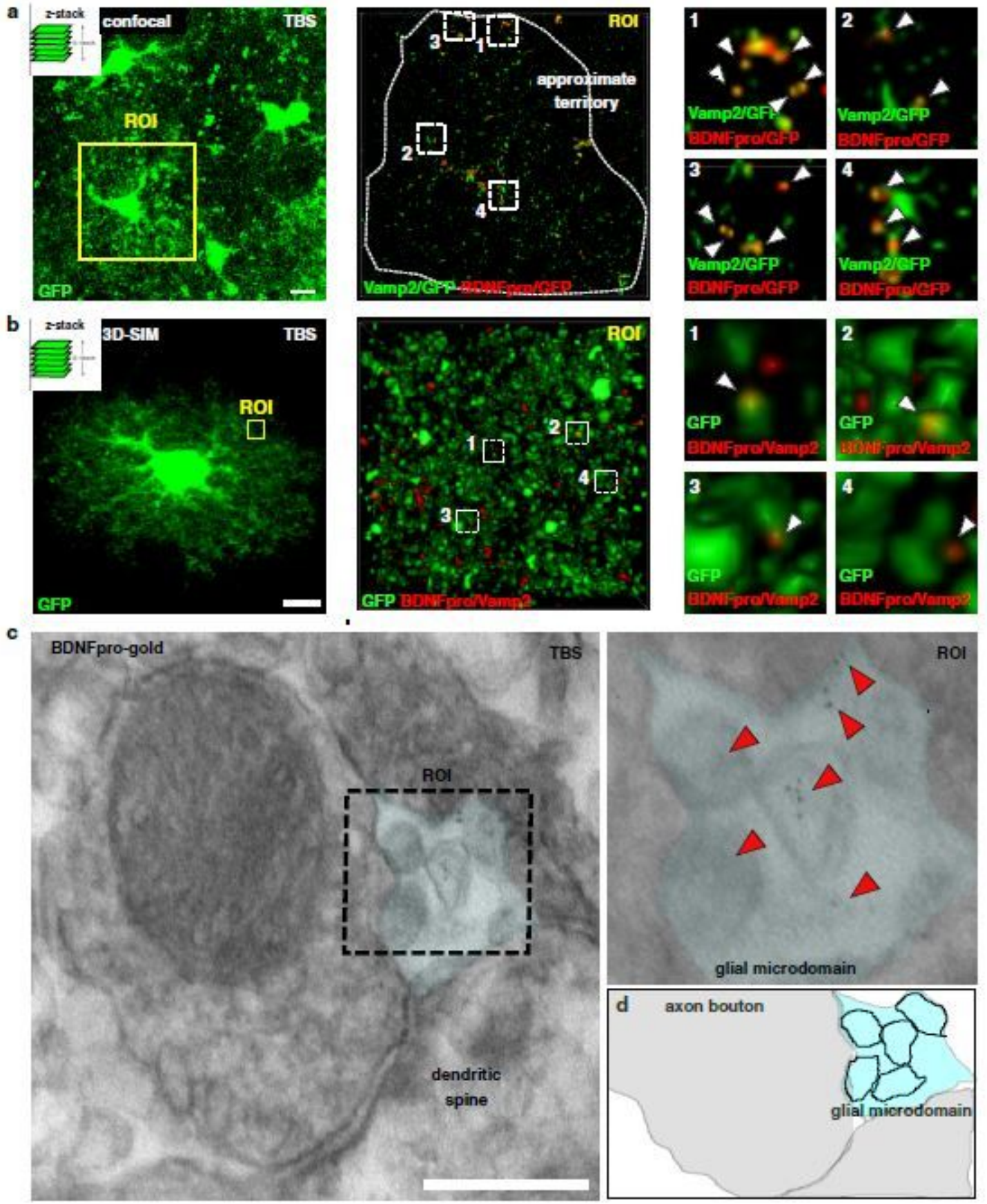

Figure 4

vesicular localization of BDNFpro (a) z-stack reconstruction shows astrocytes labeled by GFP (left). Cortical slices from control mice injected with AAV-GFAP-GFP virus were fixed 10 min after TBS and 
processed for immunostaining and confocal analysis. Three times magnification of a ROI shows one GFP-astrocyte delimited by an approximate territory (white dashed) (middle). BDNFpro/GFP and Vamp2/GFP co-localizations signals are shown. Seven times magnification shows representative areas (dashed square 1 to 4) in which BDNFpro/GFP and Vamp2/GFP signals overlap (right). Scale bars: $5 \mu \mathrm{m}$. (b) 3D-SIM image of a GFP-labeled astrocyte in a TBS-slice from control mice (left). Twenty times magnification of a ROI (middle) in which BDNFpro/Vamp2 colocalization signal is shown. Seven times magnification shows BDNFpro/Vamp2 colocalization signal in fine membrane extensions of the cell periphery (dashed square 1 to 4) (right). Scale bar: $5 \mu \mathrm{m}$. (c) EM image depicts BDNFpro-gold at astrocytic microdomains (light blue) surrounding an axon bouton (left). Three times magnification of the ROI shows gold particles (red arrowheads) in vesicular-like structures (right). Scale bar: $100 \mathrm{~nm}$. (d) Digital reconstruction of the image in (c). Astrocytic vesicles (black boundary) are shown. Scale bar: 100 $\mathrm{nm}$. 
a

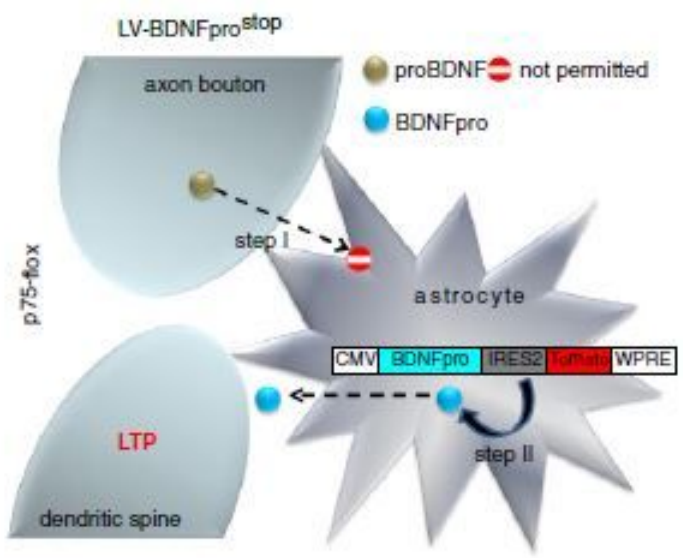

b
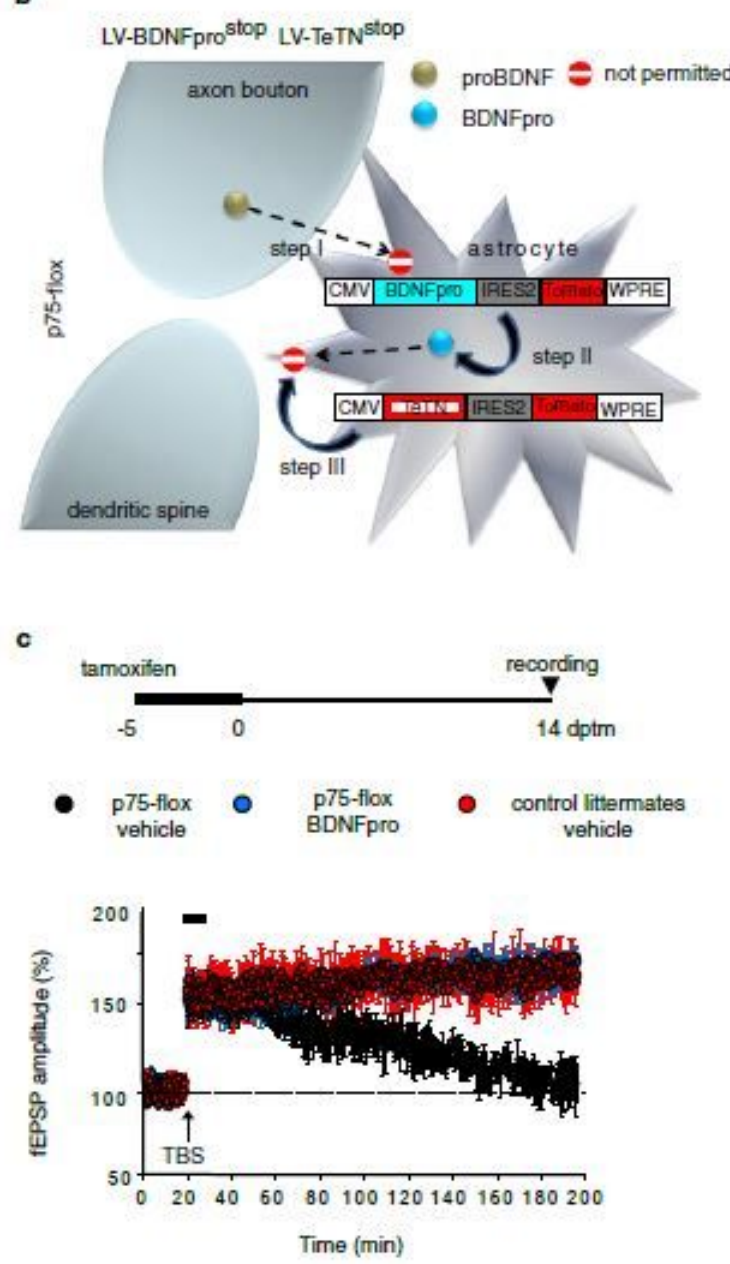
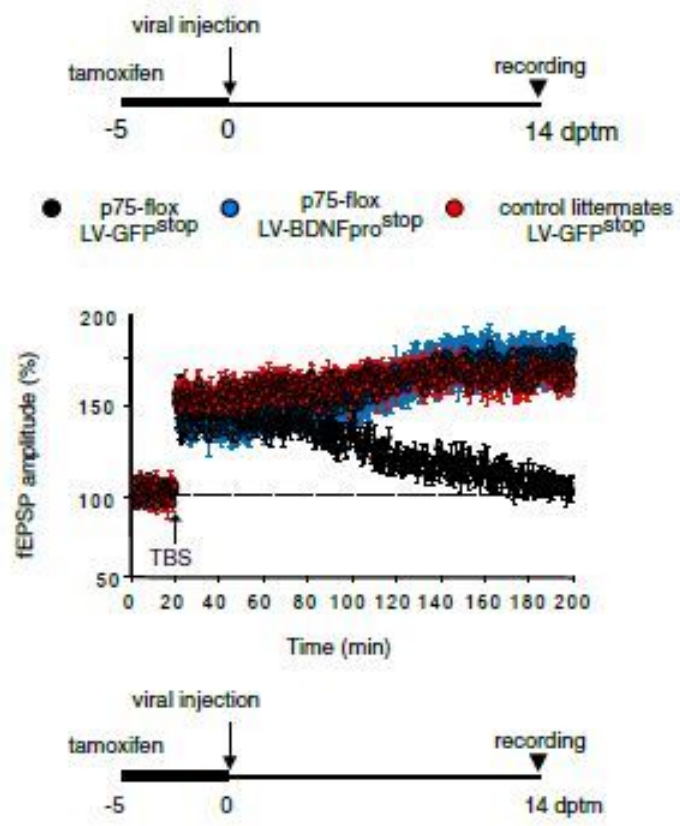

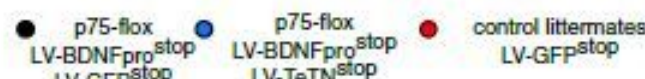

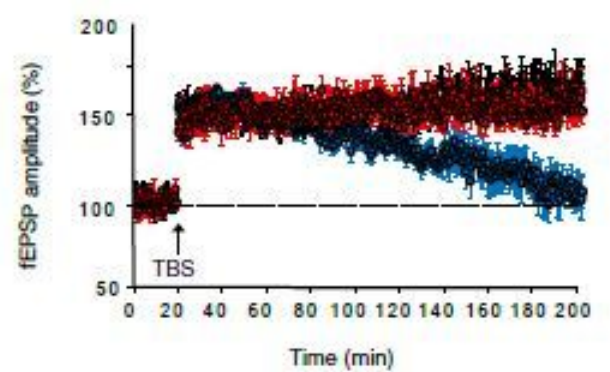

d
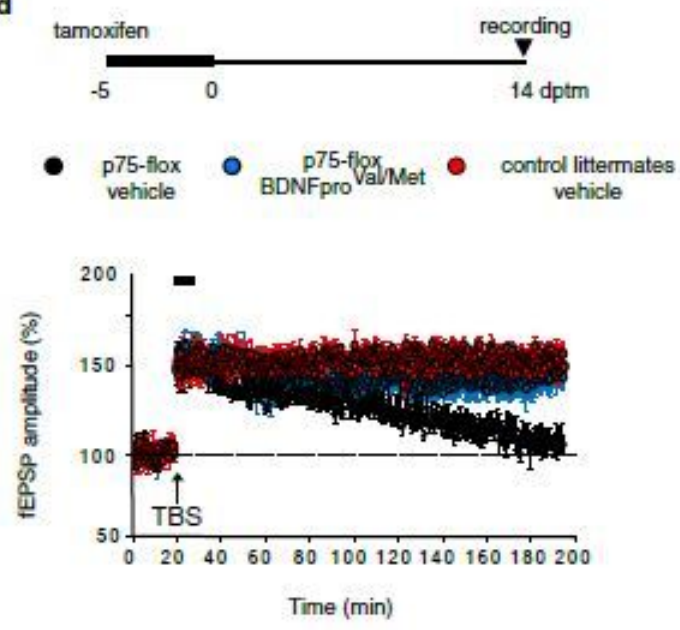

\section{Figure 5}

astrocytic BDNFpro secretion rescues LTP deficit in p75-flox mice (a) Schematic representation of the experimental design (left). Step I, deletion of p75NTR in astrocytes from tamoxifen-treated p75-flox mice precludes proBDNF transfer from neurons to astrocyte following TBS. Step II, LV-BDNFprostop transduction replaces BDNFpro in astrocytes. Schematic representation of the experimental paradigms (right); mice were treated with tamoxifen (-5 to 0$)$, injected with lentiviruses the last day of tamoxifen 
treatment $(0 \mathrm{dptm})$ and finally recorded $(14 \mathrm{dptm})$. LTP evoked in slices from p75-flox mice and control littermates injected with LV-GFPstop or LV-BDNFprostop is shown. Data are presented as mean \pm SEM; ( $n$ = 10 slices, 6 mice for $\mathrm{p} 75$-flox/LV-GFPstop; $\mathrm{n}=9$ slices, 6 mice for $\mathrm{p} 75$-flox/LV-BDNFprostop; $\mathrm{n}=6$ slices, 4 mice for control littermates/LV-GFPstop). (b) Schematic representation of the experimental design (left). Step II, and I as in (a). Step III, LV-TeTNstop transduction in astrocytes prevents from BDNFpro release. Schematic representation of the experimental paradigm as in (a) (right). LTP evoked in slices from p75-flox mice and control littermates injected with LV-GFPstop or co-injected with LV-GFPstop/LVBDNFprostop and LV-TeTNstop/LV-BDNFprostop is shown. Data are presented as mean \pm SEM; $(n=8$ slices, 6 mice for $\mathrm{p75}$-flox/LV-BDNFprostop/LV-GFPstop; $\mathrm{n}=9$ slices, 7 mice for $\mathrm{p} 75$-flox/LVTeTNstop/LV-BDNFprostop; $n=7$ slices, 4 mice control littermates/LV-GFPstop). (c) LTP evoked in slices from p75-flox mice and control littermates. Mice were treated with tamoxifen ( -5 to 0$)$ and recorded 14 $\mathrm{dptm}$. Slices were perfused (18-28 $\mathrm{min}$ ) with vehicle or exogenous BDNFpro. Data are presented as mean \pm SEM; ( $n=8$ slices, 4 mice for $p 75$-flox/vehicle; $n=7$ slices, 5 mice for $p 75$-flox/BDNFpro; $n=7$ slices, 5 mice for control littermates/vehicle). (d) LTP evoked as in (c). Slices were perfused (18-28 min) with vehicle or BDNFproVal66Met. Data are presented as mean \pm SEM; $(n=8$ slices, 5 mice for $p 75-$ flox/vehicle; $n=6$ slices, 4 mice for $p 75$-flox/BDNFproVal66Met; $n=7$ slices, 5 mice for control littermates/vehicle). 


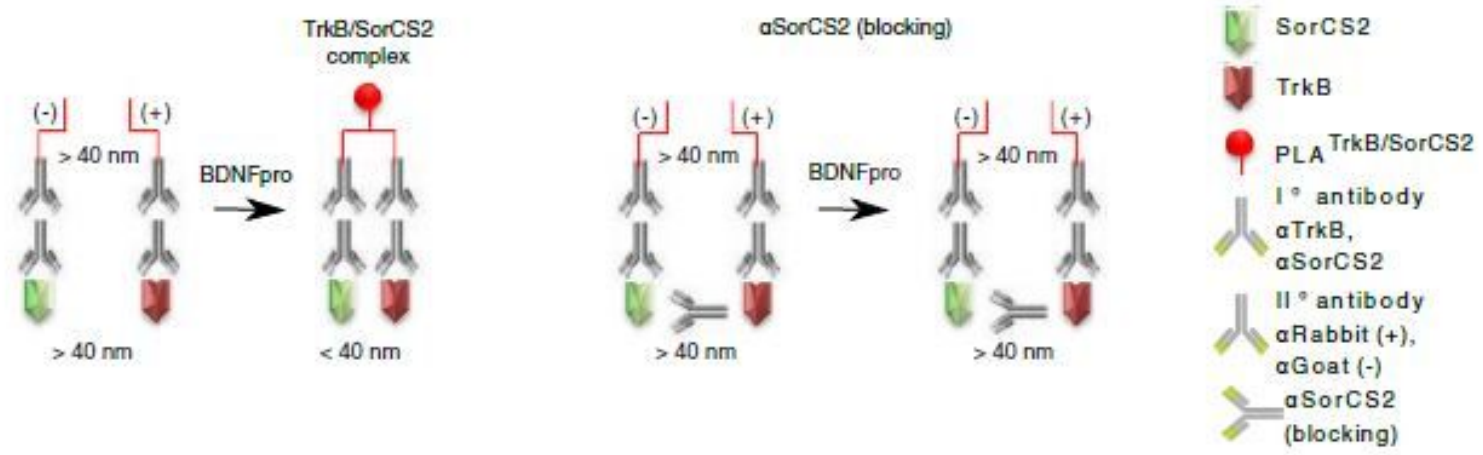

b
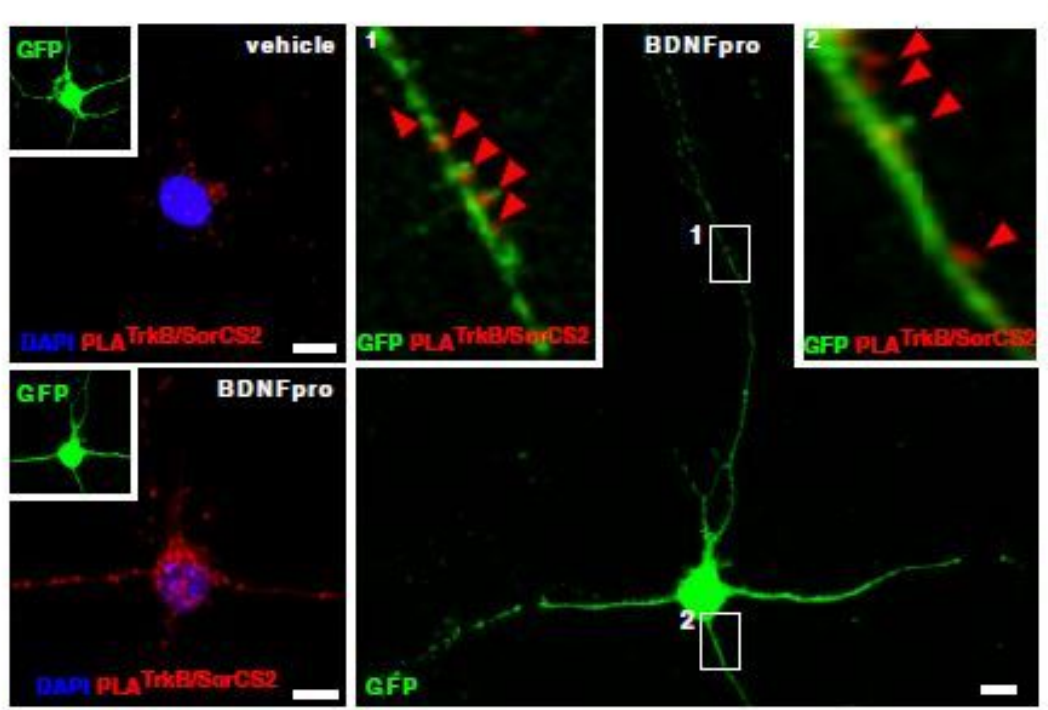

c
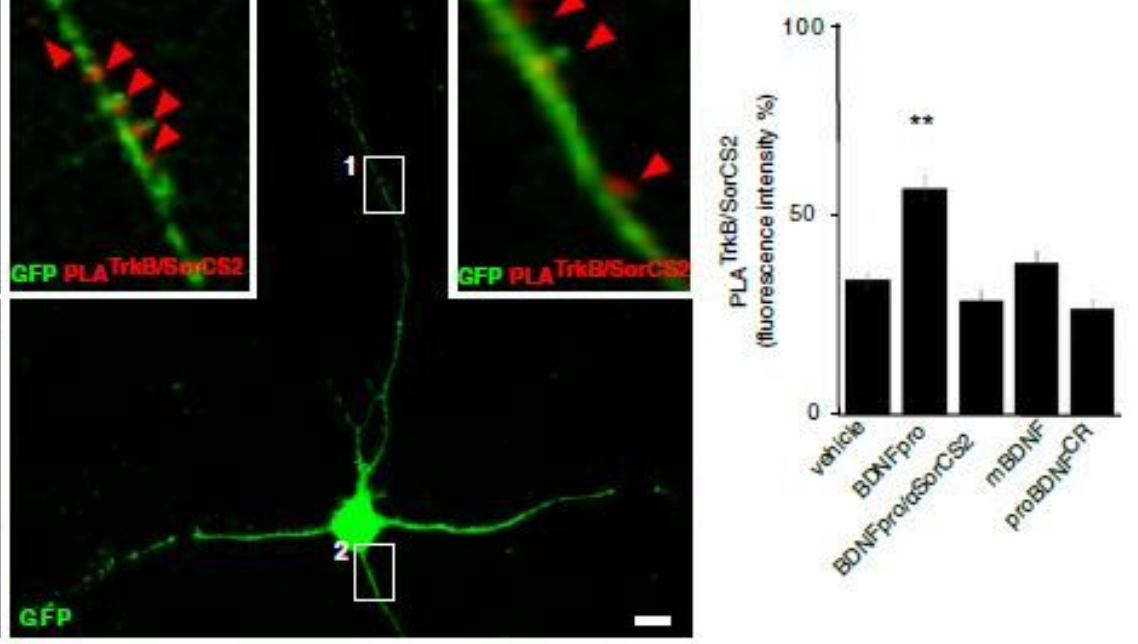

d
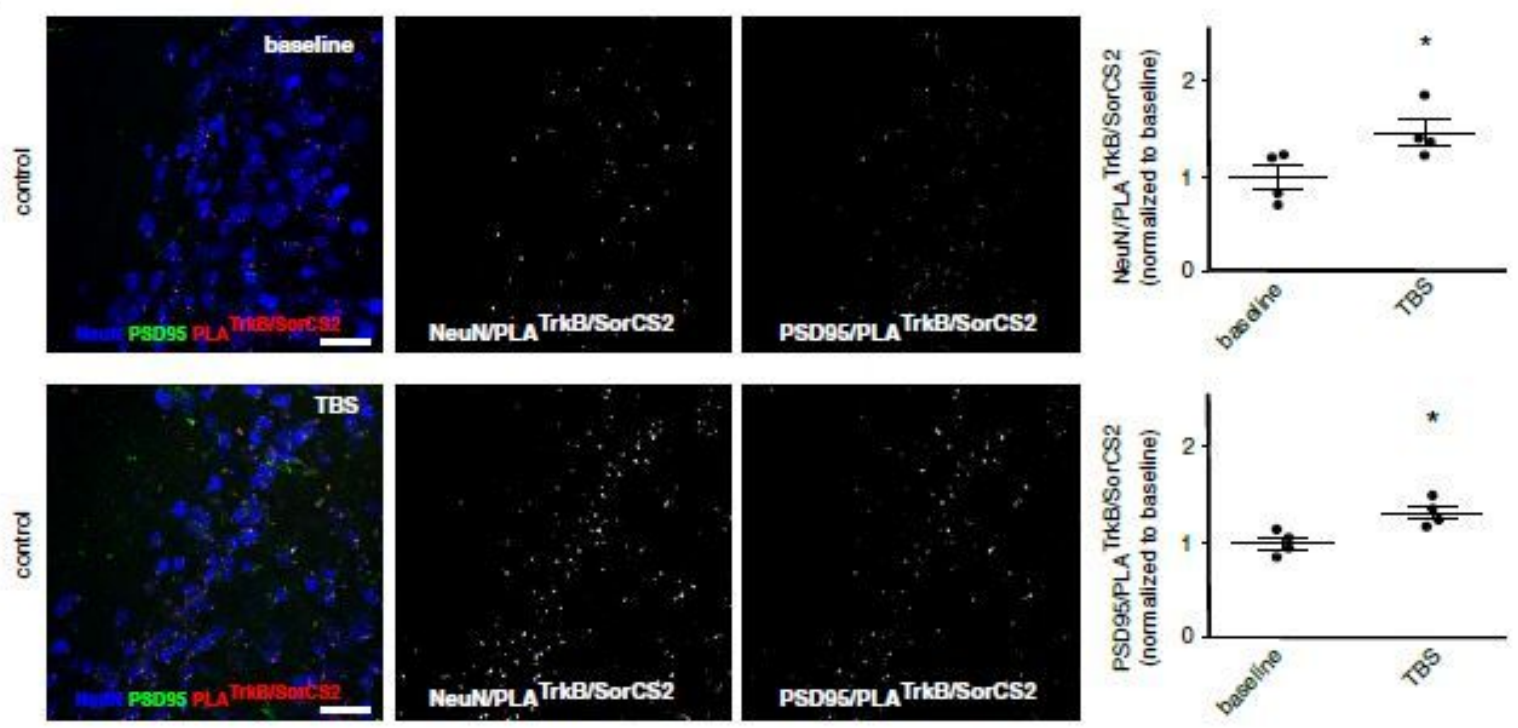

\section{Figure 6}

post-synaptic targeting of TrkB/SorCS2 complex (a) Schematic representation of the experimental design. Circular DNA probes $(-)$ and $(+)$ are coupled to $I^{\circ}$ antibody targeting aSorCS2 and aTrkB $I^{\circ}$ antibody. BDNFpro induces TrkB/SorCS2 complex formation (PLATrkB/SorCS2) that is prevented in the presence of aSorCS2 (blocking) antibody. (b) Left panel shows PLATrkB/SorCS2 signals in primary culture of cortical neurons treated with vehicle (upper) or BDNFpro (lower). The insets show reference 
GFP-neurons. Right panel shows a GFP-neuron treated with BDNFpro. Five times magnification of regions of interest 1 and 2 shows dendritic PLATrkB/SorCS2 localization (red arrowheads). Scale bars: $5 \mu \mathrm{m}$. (c) Quantification of PLATrkB/SorCS2 signal in cultured neurons treated with vehicle, BDNFpro (in presence or absence of aSorCS2), mBDNF or proBDNFCR. Data are presented as mean \pm SEM; ** $p<0.01$ (Unpaired t-test); $(n=111$ cells, 3 cultures for vehicle; $n=152$ cells, 4 cultures for BDNFpro; $n=98$ cells, 3 cultures for BDNFpro/aSorCS2; $\mathrm{n}=89$ cells, 3 cultures for mBDNF; $\mathrm{n}=79$ cells, 3 cultures for proBDNFCR). (d) $z$-stack reconstruction (left) showing NeuN, PSD95 and PLATrkB/SorCS2 signals in baseline (upper) and TBS (lower) slices. NeuN/PLATrkB/SorCS2 (center) and PSD95/PLATrkB/SorCS2 (right) colocalization signal is shown. Scale bars: $40 \mathrm{~mm}$. NeuN/PLATrkB/SorCS2 and PSD95/PLATrkB/SorCS2 colocalization was quantified using Mander's overlap. Data are normalized to baseline and presented as mean $\pm \mathrm{SEM}$; * $\mathrm{p}<0.05$ (Unpaired t-test); $(\mathrm{n}=4$ slices, 3 mice for each experimental condition). 
a
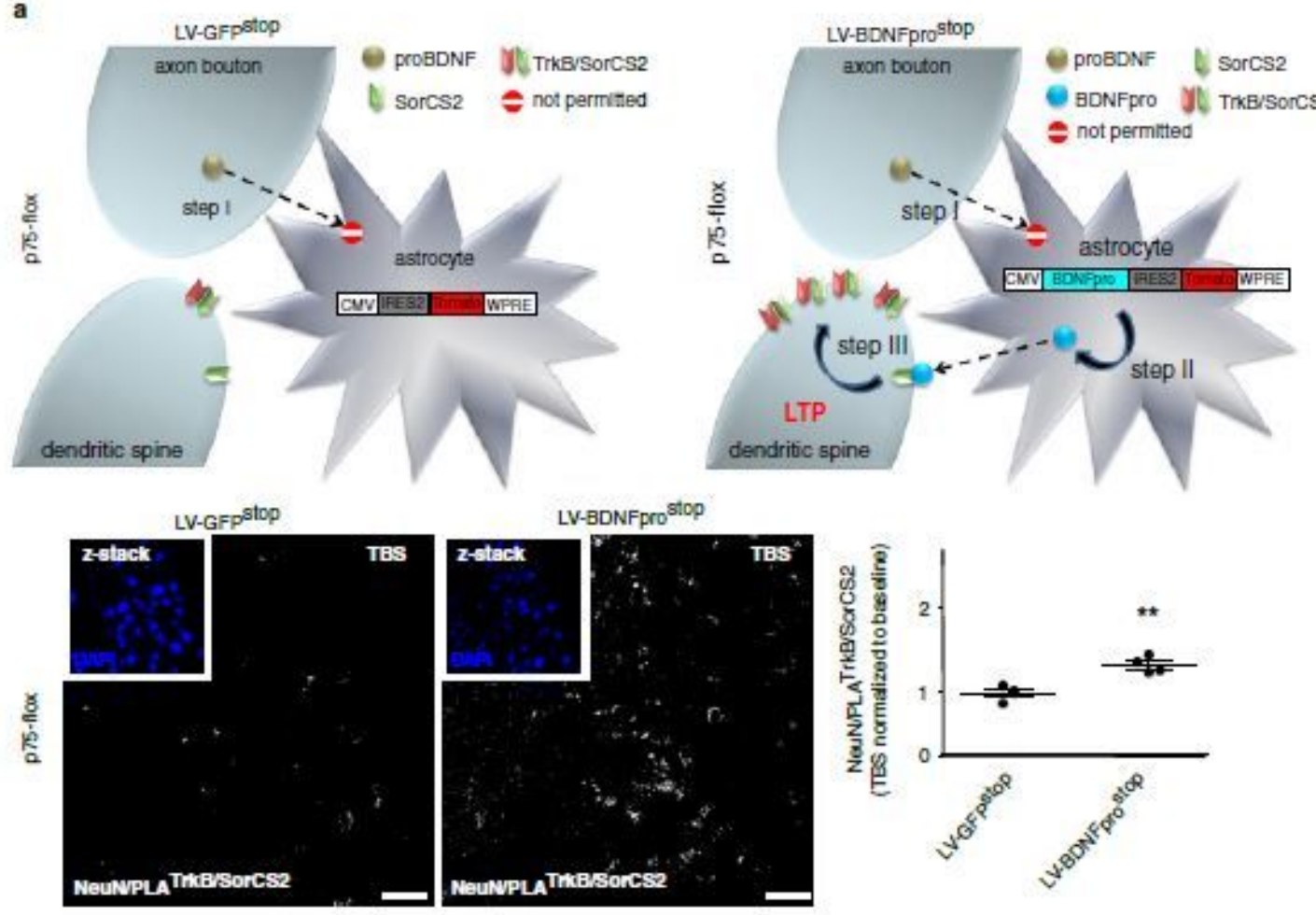

b
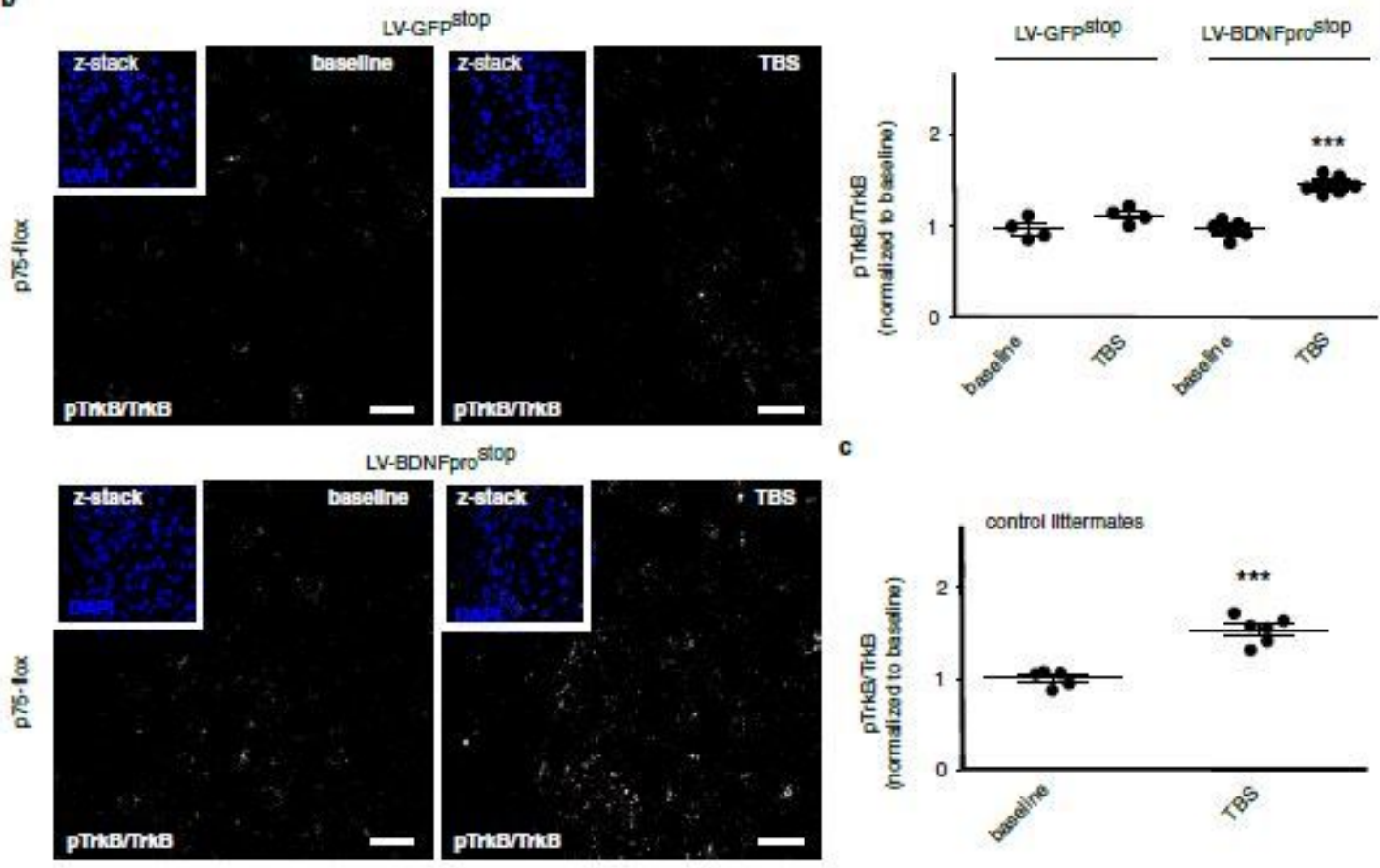

c

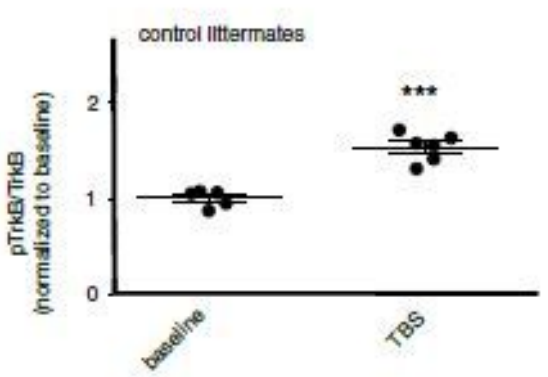

\section{Figure 7}

BDNFpro- induced TrkB/SorCS2 targeting and TrkB phosphorylation (a) Schematic representation of the experimental design (upper). Step I, deletion of p75NTR in astrocytes from tamoxifen-treated p75-flox mice precludes proBDNF transfer from neurons to astrocyte following TBS. Step II, LV-BDNFprostop transduction replaces BDNFpro in astrocytes. Step III, astrocytic BDNFpro provides final increase of TrkB/SorCS2 complexes in dendritic spines and LTP maintenance. $z$ - stack reconstruction showing 
NeuN/PLATrkB/SorCS2 colocalization signal in TBS-slices transduced with LV-GFPstop or LVBDNFprostop (lower). The insets show field of analysis. NeuN/PLATrkB/SorCS2 colocalization was quantified using Mander's overlap. Data are normalized to baseline and presented as mean $\pm \mathrm{SEM}$; ** $p<0.01$ (Unpaired t-test); $(n=4$ slices, 3 mice for each experimental condition). Scale bars: $40 \mu \mathrm{m}$ (b) zstack reconstruction showing $\mathrm{pTrkB} /$ TrkB colocalization signal in baseline- and TBS-slices from p75-flox mice transduced with LV-GFPstop (upper) or LV-BDNFprostop (lower). The insets show field of analysis. pTrkB/TrkB colocalization was quantified using Mander's overlap. Data are normalized to baseline and presented as mean $\pm \mathrm{SEM}$; $* \star \star \mathrm{p}<0.001$ (Unpaired t-test); $(\mathrm{n}=4$ slices, 3 mice for $\mathrm{p} 75$-flox mice/LVGFPstop/TBS; $n=4$ slices, 3 mice for $p 75$-flox mice/LV-GFPstop/baseline; $n=6$ slices, 4 mice for $p 75$-flox mice/LV-BDNFprostop/TBS; $n$ = 6 slices, 4 mice for p75-flox mice/LV-BDNFprostop/baseline). Scale bars: $40 \mu \mathrm{m}$. (c) Quantification of pTrkB/TrkB colocalization in baseline- and TBS-slices from control littermates using Mander's overlap. Data are normalized to baseline and presented as mean \pm SEM; $* * \star$ $p<0.001$ (Unpaired t-test); ( $n=5$ slices, 3 mice for control littermates/TBS; $n=6$ slices, 3 mice for control littermates/baseline).

a
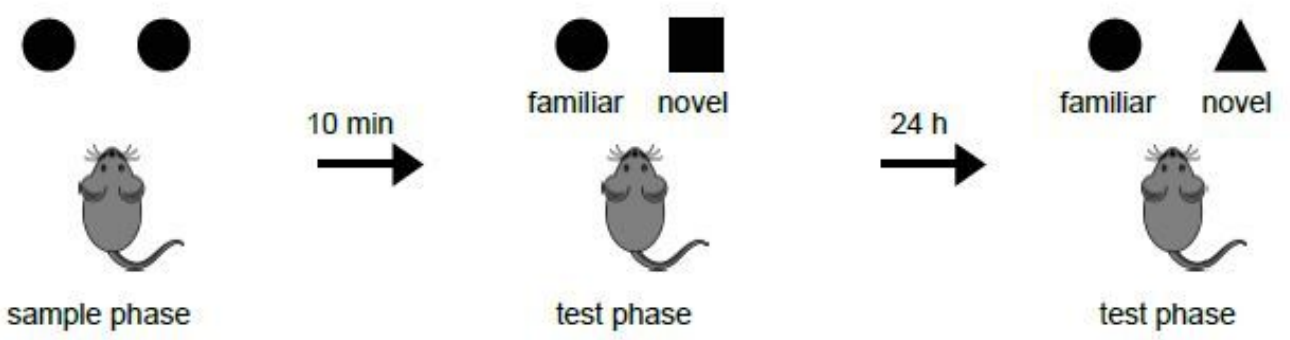

b
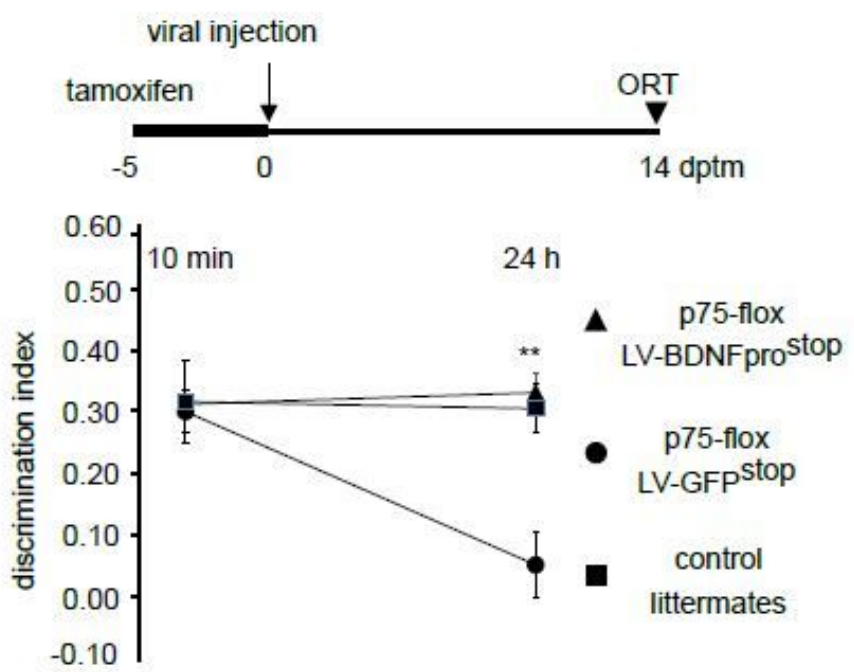

c viral injection
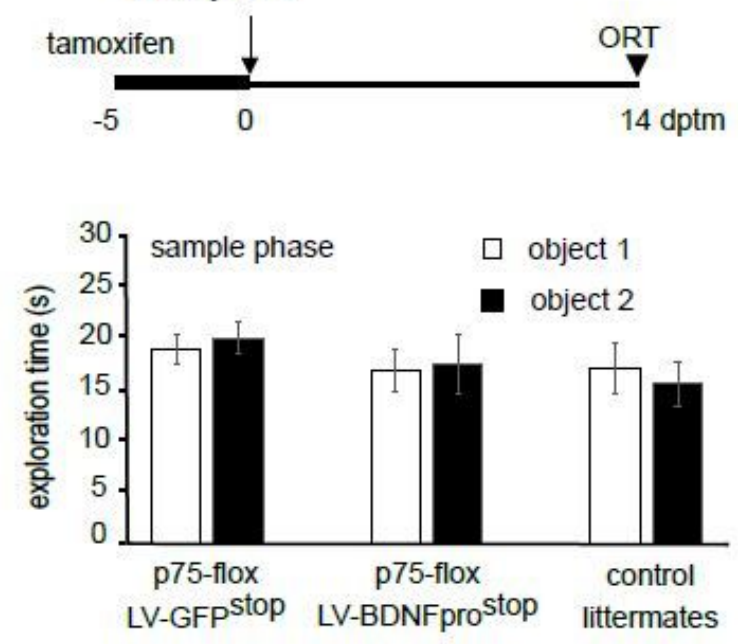

\section{Figure 8}


BDNFpro restores memory retention in p75-flox mice (a) Schematic diagram depicting the behavioral paradigm used for ORT. Mice were subjected to familiarization (sample phase) with two identical objects (circles). A test phase in which one familiar object (circle) is substituted with a novel one was performed after $10 \mathrm{~min}$ (square) and $24 \mathrm{~h}$ (triangle). (b) Schematic diagram depicting the experimental paradigm (upper). p75-flox mice and control littermates treated with tamoxifen ( -5 to 0$)$ and injected with LVGFPstop or LV-BDNFprostop the last day of tamoxifen treatment $(0 \mathrm{dptm})$ were subjected to ORT (14 $\mathrm{dptm}$ ). Discrimination index is plotted against time interval between sample phase and test phases. Data are presented as mean $\pm \mathrm{SEM}$; ${ }^{*} \mathrm{p}<0.01$ (post hoc Holm-Sidak); $(n=11$ mice for $\mathrm{p} 75$-flox/LV-GFPstop; $n$ $=10$ mice for $p 75$-flox/LV-BDNFprostop; $n=5$ mice for control littermates). (c) Schematic diagram depicting the experimental paradigm as in (b). The histogram shows mean exploration time of the familiar object and the novel object in the sample phase. Data are presented as mean \pm SEM; $(n=11$ mice for $\mathrm{p} 75$-flox/LV-GFPstop; $\mathrm{n}=10$ mice for $\mathrm{p} 75$-flox/LV-BDNFprostop; $\mathrm{n}=5$ mice for control littermates).

\section{Supplementary Files}

This is a list of supplementary files associated with this preprint. Click to download.

- Supplementaryfiguresandlegends.pdf 